2-4-2013

\title{
Microkinetic Modeling and Analysis of Ethanol Partial Oxidation and Reforming Reaction Pathways on Platinum at Short Contact Times
}

Maura A. Koehle

makoehle@gmail.com

\section{Recommended Citation}

Koehle, Maura A., "Microkinetic Modeling and Analysis of Ethanol Partial Oxidation and Reforming Reaction Pathways on Platinum at Short Contact Times" (2013). Master's Theses. 382.

https://opencommons.uconn.edu/gs_theses/382 
Microkinetic Modeling and Analysis of Ethanol Partial Oxidation and Reforming Reaction Pathways on Platinum at Short Contact Times

Maura A. Koehle

B.S., Chemical Engineering, University of Pittsburgh, 2010

\author{
A Thesis \\ Submitted in Partial Fulfillment of the \\ Requirements for the Degree of \\ Master of Science \\ at the \\ University of Connecticut
}

2013 


\section{APPROVAL PAGE}

Master of Science Thesis

\section{Microkinetic Modeling and Analysis of Ethanol Partial Oxidation and Reforming Reaction} Pathways on Platinum at Short Contact Times

\section{Presented by}

Maura A. Koehle, B.S.

Major Advisor

Douglas Cooper, Ph.D.

Associate Advisor

William Mustain, Ph.D.

Associate Advisor

Ranjan Srivastava, Ph.D.

University of Connecticut

2013 


\section{ACKNOWLDGEMENTS}

There are many people without whom this thesis would not have been possible. I must thank Ashish Mhadeshwar for the incredible amount of knowledge he imparted and the guidance he provided in order for this research to be conducted, shared at conferences and published. Many thanks to Doug Cooper, Bill Mustain and Ranjan Srivastava for standing in as advisors and providing support that was above and beyond expectations. I want to thank everyone that has become part of my UConn family, without whom this project would have been possible but much less fun: Angie Moreno, Phil Baker, Pete Menard, Leah Winterberger, Andrea Kadilak, Aida Ghiaei and the rest of the GK-12 group. Finally, I am incredibly grateful for my family and friends and their unending support. This is dedicated to my parents. 


\section{TABLE OF CONTENTS}

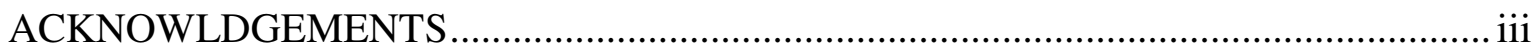

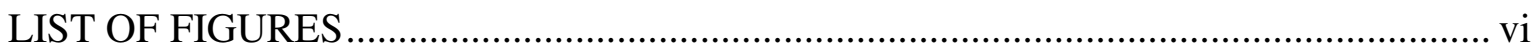

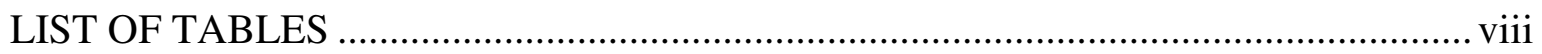

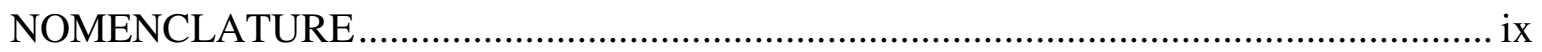

ABBREVIATIONS AND ACRONYMS ...................................................................

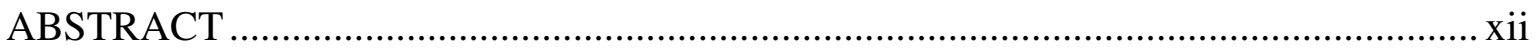

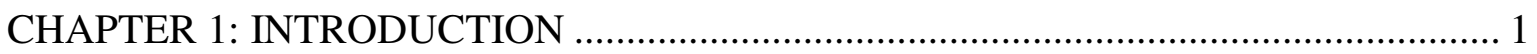

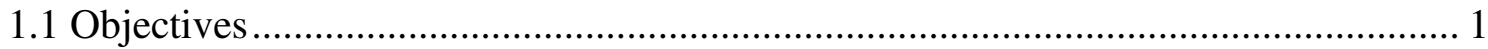

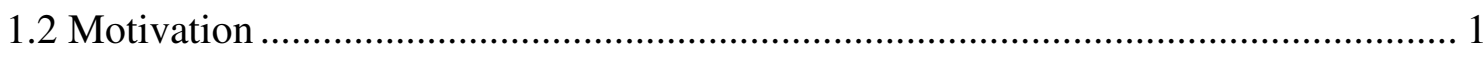

CHAPTER 2: Mechanistic Investigations for Ethanol Reforming ........................................ 4

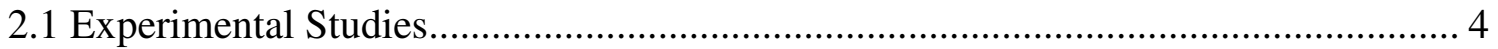

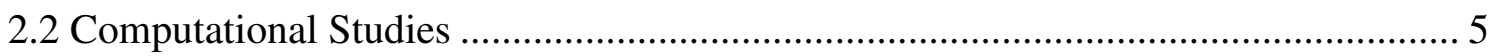

CHAPTER 3: Surface Reaction Mechanism Development ................................................. 7

3.1 Steps in the Mechanism Development ............................................................... 7

3.2 Network of Species and Elementary Surface Reactions ......................................... 9

3.3 Kinetic Parameter Estimation................................................................................ 16

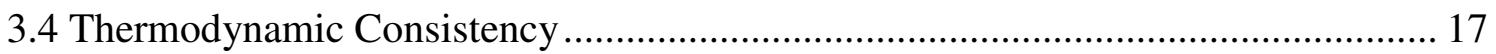

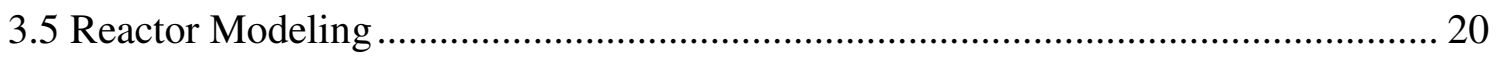

3.6 Model Assumptions and Limitations .................................................................... 20

CHAPTER 4: Performance and Optimization of the Surface Reaction Mechanism.......... 23

4.1 Identification and Refinement of Important Parameters ............................................ 24

4.2 Mechanism Predictions against Experimental Data.................................................. 27

CHAPTER 5: Mechanistic Analysis and Reaction Zones ................................................. 28

5.1 Zone 1: Ethanol Dehydration and Oxidation .......................................................... 30

5.2 Zone 2: Transition: Ethanol Oxidation and Dry Reforming ...................................... 33

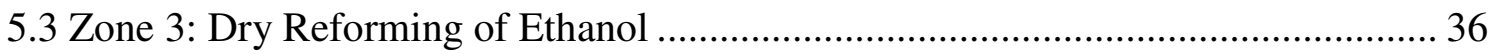

5.4 Zone 4: Dry and Steam Reforming of Methane ………………………………....... 39

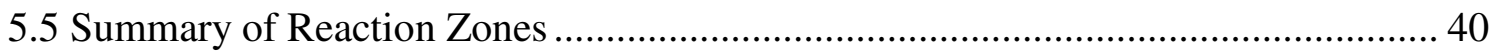

5.6 Reaction Order Analysis ..................................................................................... 43

CHAPTER 6: Mechanism Validation against Dry Reforming Experiments ....................... 44 
6.1 BenchCAT Fixed Bed Reactor Experiments.................................................. 44

6.2 Mechanism Validation for Dry Reforming of Methane ........................................ 45

6.3 Mechanistic Analysis and Dominant Reaction Pathways ..................................... 48

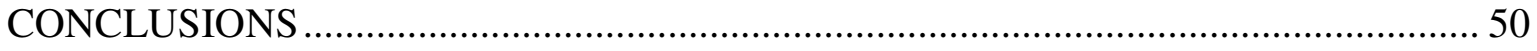

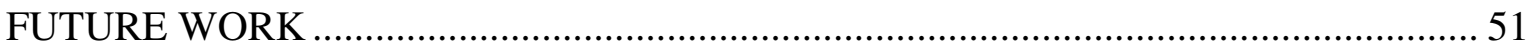

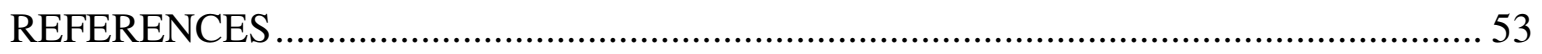




\section{LIST OF FIGURES}

Figure 3.1: $\quad$ Surface Reaction Mechanism Development............................8

Figure 3.2: Ethanol Reforming Reaction Mechanism..............................10

Figure 3.3: $\quad \mathrm{K}_{\mathrm{eq}}$ Ratio $\left(\mathrm{K}_{\mathrm{eq}}{ }^{\text {surface }} / \mathrm{K}_{\mathrm{eq}}{ }^{\mathrm{gas}}\right)$ for Surface Reaction Mechanisms..............18

Figure 4.1: Comparison of Model Simulation to Experiment......................24

Figure 4.2: Model Sensitivity Analysis........................................25

Figure 5.1: $\quad$ Simulated Mole Fraction Profiles for Ethanol Partial Oxidation.............29

Figure 5.2: $\quad$ First Zone Primary Surface Reaction Pathways: Ethanol Dehydration and

Oxidation.......................................................... 31

Figure 5.3: First Zone Primary Gas Phase Reaction Pathways: Ethanol Dehydration and Oxidation...................................................... 32

Figure 5.4: Second Zone Primary Surface Reaction Pathways: Transition Between

Ethanol Oxidation and Dry Reforming............................... 34

Figure 5.5: Second Zone Primary Gas Phase Reaction Pathways: Transition Between

Ethanol Oxidation and Dry Reforming..................................35

Figure 5.6: Third Zone Primary Surface Reaction Pathways: Dry Reforming of

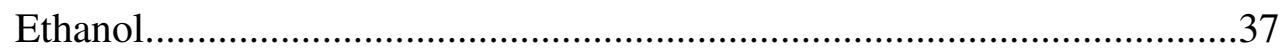

Figure 5.7: Third Zone Primary Gas Phase Reaction Pathways: Dry Reforming of

Ethanol........................................................ 38

Figure 5.8: $\quad$ Fourth Zone Primary Surface Reaction Pathways: Dry and Steam

Reforming of Methane..............................................40 
Figure 5.9: Simulated Coverage Profiles for Ethanol Partial Oxidation................41

Figure 5.10: Reaction Zone Distribution at Various Reaction Temperatures............42

Figure 6.1: Experimental Setup for Methane Dry Reforming Experiments............45

Figure 6.2: Mechanism Validation Against Methane Dry Reforming Experimental

Data........................................................46

Figure 6.3: Comparison of Model Predicted and Experimental Mole Fractions for Methane Dry Reforming.........................................47

Figure 6.4: Primary Reaction Pathways for Methane Dry Reforming.................49 


\section{LIST OF TABLES}

Table 1: $\quad$ Surface Reaction Mechanism for Ethanol Reforming on Pt................11

Table 2: $\quad$ Species Binding Energies, Coverage and Temperature Dependence

Coefficients...................................................... 


\section{NOMENCLATURE}
A
$\mathrm{S}^{-1}$
Pre-exponential factor

$\mathrm{A}_{\mathrm{c}} / \mathrm{V}_{\mathrm{R}} \quad \mathrm{cm}^{-1} \quad$ Catalyst area per unit reaction volume

d

$\mathrm{cm}$

Pore or tube diameter

E

$\mathrm{kcal} / \mathrm{mol}$

Activation energy

G

$\mathrm{mol} / \mathrm{cm}^{3} \cdot \mathrm{s} \quad$ Rate of gas phase species

M

$\mathrm{g} / \mathrm{mol}$

Molecular weight of species

Q

$\mathrm{kcal} / \mathrm{mol}$

Binding energy

$\mathrm{R}$

$\mathrm{erg} / \mathrm{mol} / \mathrm{K}$

Universal gas constant

$\mathrm{s}$

unitless

Sticking coefficient

S

$\mathrm{mol} / \mathrm{cm}^{2} \cdot \mathrm{s} \quad$ Rate of surface species

$\Delta \mathrm{S}$

$\mathrm{erg} / \mathrm{mol} / \mathrm{K} \quad$ Entropy of reaction

$\mathrm{T}$

K

Temperature

$\mathrm{u}$

$\mathrm{cm} / \mathrm{s}$

Velocity

Y

unitless

Mass fraction of species

Z

$\mathrm{cm}$

Length

$\alpha$

$\mathrm{kcal} / \mathrm{mol} / \mathrm{ML}$

Coverage dependence coefficient

$\beta$

unitless

Modified Arrhenius equation temperature exponent

$\gamma$

unitless

Temperature dependence coefficient for binding energies

$\theta$

unitless

Coverage of species

$\rho$

$\mathrm{g} / \mathrm{cm}^{3}$

Mass density

$\sigma$

$\mathrm{mol} / \mathrm{cm}^{2}$

Site density

Subscript k

Species number 
Subscript $\mathrm{f}$

Subscript b
Forward reaction

Backward reaction 


\section{ABBREVIATIONS AND ACRONYMS}

$\begin{array}{ll}\text { DFT } & \text { Density Functional Theory } \\ \text { MARI } & \text { Most Abundant Reaction Intermediate } \\ \mathrm{Pt} & \text { Platinum } \\ \text { RPA } & \text { Reaction Pathway Analysis } \\ \text { RWGS } & \text { Reverse Water Gas Shift } \\ \text { UBI-QEP } & \text { Unity Bond Index-Quadratic Exponential Potential } \\ \text { WGS } & \text { Water Gas Shift }\end{array}$




\section{ABSTRACT}

Catalytic reforming of biomass-derived ethanol is a promising pathway for essentially carbon-neutral hydrogen production. The feasibility and performance of this process has been demonstrated over the last several years, especially at short contact times. Despite the extensive experimental work conducted in this area, there remains a need for a comprehensive kinetic model for ethanol reforming that will facilitate detailed reactor design, rational catalyst design, and process development. In this work, a novel microkinetic model for ethanol partial oxidation and reforming on Platinum $(\mathrm{Pt})$, consisting of 100 irreversible elementary steps, is developed and validated using experimental data at short contact times. Kinetic parameters are taken from quantum mechanical Density Functional Theory (DFT) calculations reported in literature, extracted from surface science experimental data, or estimated using semi-empirical and firstprinciples methods. The microkinetic model is thermodynamically consistent at both the enthalpic and entropic levels. Depending on the chosen gas phase reaction mechanism and as guided by sensitivity and reaction path analyses, seven kinetic parameters are adjusted to improve the model performance against literature experimental data for ethanol reforming. Species coverage and mole fraction profiles as well as reaction pathways and overall rates are analyzed to gain a fundamental understanding of the variations in the dominant catalytic chemistry on Pt with changing temperature. Our analysis indicates the presence of up to 4 reaction zones over the length of the catalyst, viz., (i) dehydration and complete oxidation of ethanol, (ii) transition between oxidation and dry reforming of ethanol, (iii) dry reforming of ethanol, and (iv) steam and dry reforming of methane. As the majority of the catalytic zones are dominated by dry reforming conditions, additional validation of the microkinetic model is carried out with methane dry reforming 
experiments on $\mathrm{Pt} / \mathrm{Al}_{2} \mathrm{O}_{3}$. The proposed microkinetic model for ethanol partial oxidation and reforming is able to predict the dry reforming data without any further adjustment of the kinetic parameters.

Keywords: Ethanol, oxygenates, partial oxidation, reforming, platinum, microkinetic model, reaction pathways 


\section{CHAPTER 1: INTRODUCTION}

\subsection{Objectives}

The purpose of this work was to develop and validate a microkinetic model for ethanol partial oxidation and reforming on Platinum. Such a model allows for reaction pathway analysis and detailed understanding of the chemistry on the catalytic surface.

\subsection{Motivation}

Success of the hydrogen $\left(\mathrm{H}_{2}\right)$ economy and widespread implementation of $\mathrm{H}_{2}$ as the fuel of the future requires significant technological advances in $\mathrm{H}_{2}$ production, transportation, storage, utilization, and carbon dioxide $\left(\mathrm{CO}_{2}\right)$ capture. Processing of conventional fuels (e.g., reforming of natural gas) for $\mathrm{H}_{2}$ production is associated with net $\mathrm{CO}_{2}$ emissions, which is a potent greenhouse gas. Fuel processing at a centralized location may allow $\mathrm{CO}_{2}$ capture and sequestration (CCS), provided cost-effective technologies are developed for CCS. Alternatively, $\mathrm{H}_{2}$ could be obtained from renewable and sustainable sources such as biomass, in order to reduce net $\mathrm{CO}_{2}$ emissions. During the growth of biomass, plants consume $\mathrm{CO}_{2}$ from the atmosphere and convert it into carbohydrates via photosynthesis. It is then released back into the atmosphere via reforming of biomassderived chemicals (e.g. oxygenates such as ethanol, glycerol, biodiesel, and bio-oil), thereby resulting in a closed carbon cycle [1].

Biomass-derived ethanol is a promising renewable energy source that has been developed and implemented in several countries, such as the United States and Brazil, in the latter with great success. A major drawback of bio-ethanol fuel, however, is its high water content, which must be separated using expensive distillation and zeolite adsorption 
processes [1]. An alternative to using bio-ethanol directly as a fuel is to reform it to yield hydrogen. Bio-ethanol may also be reformed in a variety of ways, including reaction with water (steam reforming), oxygen (partial oxidation), carbon dioxide (dry reforming), or combinations of the three [2]. Such reforming can also yield syngas, which can be used in fuel cells or upgraded through the Fischer-Tropsch process to produce synthetic fuels and high value chemicals. Syngas production is likely to be a more immediate application of bio-ethanol reforming.

Extensive research has been carried out in the field of biomass-derived oxygenates reforming, especially in the discovery, design, and testing of suitable catalytic materials as well as process development (e.g., see review papers [2-12]). However, a comprehensive understanding of the underlying chemical kinetics is still lacking at an elementary reaction level, due to the complexity of oxygenates reforming chemistry. The generation of reliable, predictive, and comprehensive kinetic models will assist in chemical reactor design, process optimization, and systematic assessment of chemical processes that utilize such oxygenates.

In this work, we propose a novel and comprehensive microkinetic model for ethanol partial oxidation and reforming on Platinum $(\mathrm{Pt})$ that provides a fundamental understanding of the catalytic reaction chemistry. To our knowledge, this is the first time that a comprehensive reaction mechanism has been developed for ethanol partial oxidation and reforming on $\mathrm{Pt}$, using a hierarchical multiscale modeling approach[13-17]. The manuscript is organized as follows. Chapter 2 provides a brief background of the experimental and computational mechanistic studies for ethanol partial oxidation and reforming. The approach for microkinetic model development is described in Chapter 3. 
Chapter 4 focuses on the identification and optimization of kinetic parameters, and model performance against experimental data. Mechanistic analysis of species profiles, reaction zones, and reaction order is presented in Chapter 5. Additional validation of the microkinetic model against experiments for dry reforming of methane is presented in Chapter 6, followed by conclusions and a discussion of future work. 


\section{CHAPTER 2: Mechanistic Investigations for Ethanol Reforming}

\subsection{Experimental Studies}

Ethanol reforming processes, such as steam reforming, partial oxidation, and dry reforming, have been studied experimentally on various transition metal catalysts such as $\mathrm{Pt}$ [18-22], Rh [22-27], Ru [28], Co [29-31], and Ni [32], as well as bimetallics such as Rh$\mathrm{Ru}$ [22] and Rh-Pt [23]. Noble metals are well studied as they are not as susceptible to deactivation, but less expensive catalysts such as $\mathrm{Ni}$ and $\mathrm{Co}$ are also studied in the interest of economy. The Deutschmann and Schmidt research groups have demonstrated the feasibility of catalytic partial oxidation and reforming of ethanol at short contact times[1,22,27,33]. The Chen and Barteau research groups have used Temperature Programmed Desorption (TPD) experiments to identify the gas phase products of reforming $\left(\mathrm{H}_{2}, \mathrm{CO}\right.$, unreacted oxygenates, and $\left.\mathrm{CH}_{4}\right)$ on various catalysts $(\mathrm{Pt}, \mathrm{PtNiPt}$, NiPtPt, and Ni) [34]. Using TPD and Temperature Programmed Reaction (TPR) experiments, the Verykios research group has explored the dominant pathways for ethanol steam reforming on $\mathrm{Ni}$, such as dehydration, dehydrogenation, Water-Gas Shift (WGS), reforming, methanation, and carbon deposition [32]. Using ultra-high vacuum TPD experiments, Vesselli et al. have explored the role of oxygen in ethanol decomposition and hydrogen formation on $\mathrm{Rh}$ [35] and suggested that an oxidizing environment is required to promote decomposition of $\mathrm{C}_{2}$-molecules and to avoid coking. TPD and TPR investigations of Benito et al. for ethanol on Co have provided valuable insights regarding the mechanistic pathways of oxygenates reforming [36]. These studies highlighted ethanol decomposition pathway dependence on support (i.e. dehydration on alumina) as well as surface coverage (i.e. water formation with high oxygen surface coverage). Main reaction products for ethanol partial oxidation on $\mathrm{Pt}$ are $\mathrm{H}_{2}, \mathrm{CO}, \mathrm{CH}_{4}, \mathrm{CO}_{2}$, and $\mathrm{H}_{2} \mathrm{O}$, while 
acetaldehyde, ethylene, and methanol have been identified as minor side products $[22,27,37,38]$. At high temperatures, ethanol conversion and $\mathrm{H}_{2}$ yield are found to increase with temperature for partial oxidation on Pt [38]. Based on the products and intermediate species, hypotheses for ethanol decomposition chemistry on Pt have been proposed, including dehydrogenation of ethanol to ethoxy [37,39], dissociative adsorption of ethanol to acetaldehyde [26], or ethanol dehydration to ethylene $[18,40]$. These findings were taken into account when developing the elementary reaction pathways in the microkinetic model for ethanol partial oxidation and reforming on Pt.

\subsection{Computational Studies}

On the modeling front, a number of studies have focused on understanding the thermodynamic, mechanistic, and kinetic aspects of ethanol reforming. Thermodynamic analysis for ethanol reforming has been carried out to understand the effect of operating conditions on product selectivities and yields [41-43]. A number of studies have focused

on developing Langmuir-Hinshelwood-Hougen-Watson (LHHW) or Eley-Rideal rate expressions followed by data fitting of the kinetic parameters [44-47].

In more fundamental first-principles computational investigations, Density Functional Theory (DFT) has been used to determine species stability and reaction pathways with the lowest activation energy on various catalyst surfaces. Mavrikakis and colleagues have studied ethanol decomposition on various transition metals and proposed Bronsted-Evans-Polanyi (BEP) relationships [48]. They developed potential energy surfaces for $\mathrm{C}-\mathrm{O}$ and $\mathrm{C}-\mathrm{C}$ bond breaking in ethanol, which lead to hydrogen production and alkane production, respectively. Vlachos and colleagues have proposed a group additivity approach for predicting the oxygenate stability on metal catalysts[49]. Wang et 
$a l$. have reported dissociation barriers and heats of reactions for ethanol decomposition on a number of metal surfaces and also correlated the dissociation barriers with d-band centers[50]. Barteau and Chen research groups have reported DFT-based binding energies for some key species, such as ethanol and ethoxy, adsorbed on the $\mathrm{Ni} / \mathrm{Pt}(111)$ bimetallic surface, and have also correlated reforming reaction yields and selectivities with the surface d-band center for various catalysts (PtNiPt, Pt, Ni, and NiPt) [34,51]. The Neurock research group has reported DFT-based binding energies for a number of key species participating in $\mathrm{C}_{2}$ chemistry on $\mathrm{Pd}$ and $\mathrm{Re}$ [52]. In another DFT study of various pathways for ethanol decomposition on Pt (111), Dumesic and colleagues have reported energetics for the C-C and C-O bond cleavage in surface species [53]. Such quantum mechanical parameter estimation studies and correlations with experimental data are critical for developing detailed kinetic models and have been used as inputs in this work, as explained later.

It is also important to note that a comprehensive gas phase reaction mechanism has been developed by Marinov for high temperature ethanol oxidation [54]. This mechanism has been validated against non-catalytic empty tube experimental data for ethanol partial oxidation in the $600-900^{\circ} \mathrm{C}$ temperature range. Even though Christensen et al. found qualitative, but not quantitative, agreement with experimental data using the Marinov mechanism[55], Salge et al. found that the mechanism predicts most product selectivities within $2 \%$ [22]. We have combined the surface mechanism developed in this work with the Marinov mechanism, to accurately capture the homogeneous and heterogeneous reaction chemistry. 


\section{CHAPTER 3: Surface Reaction Mechanism Development}

The overall approach for surface reaction mechanism development was derived from microkinetic modeling concepts proposed by Dumesic and colleagues in the early 1990s [56], which was further extended by various researchers through hierarchical multiscale modeling [13-17]. Microkinetic modeling has been applied in the past for a variety of chemical processes such as methane partial oxidation and reforming $[14,57,58]$, selective catalytic reduction of $\mathrm{NO}_{x}$ [59,60], $\mathrm{NO}$ oxidation [61], ammonia decomposition[13,62], ethylene hydrogenation [63], and Water-Gas Shift [64-66], to name a few recent applications.

\subsection{Steps in the Mechanism Development}

Steps in the mechanism development are shown in Figure 3.1 and briefly summarized here. Specific details for ethanol reforming on $\mathrm{Pt}$ are discussed in the next sub-sections. The approach starts with a detailed mechanism composed of anticipated surface reactions for the considered chemical process. Kinetic parameters associated with the species and reactions are estimated using a combination of surface science experiments, semi-empirical methods, and first-principles calculations. Thermodynamic consistency is ensured at the individual reaction level as well as at the overall mechanism level using the approach described in [67]. Reactor modeling consistent with the experimental details is carried out to predict the typically measured responses, such as reactant conversion, product selectivity, species concentrations, and temperature. At this level, the predictions of the microkinetic model are typically qualitative, but may not show quantitative comparison with integral data. Such discrepancy is quite common and may arise from various uncertainties and assumptions including those related to the choice of 
reactions and species (mechanism complexity vs. practical usability), choice of kinetic parameters (accuracy of estimation methods, pressure and materials gap), experimental methods (product and intermediate species identification, availability of temperature and species concentration profiles, measurement of small values), and choice of reactor model (role of diffusion vs. kinetics). This uncertainty is inherent to model development, but provides justification for some parameter adjustment.

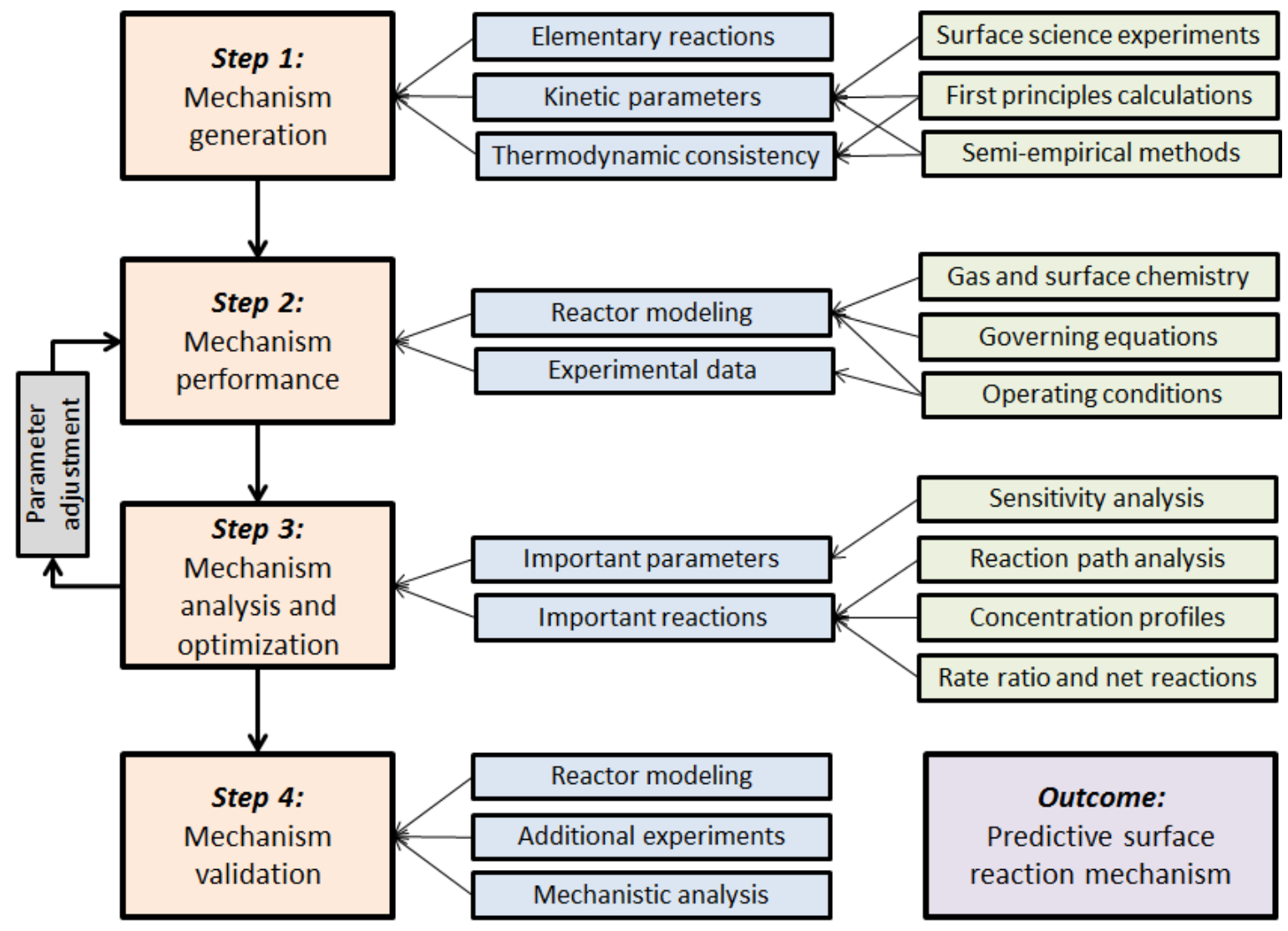

Figure 3.1: Steps in surface reaction mechanism development

Recently developed approaches in reaction kinetics, such as first-principles methods to determine all kinetic parameters [68], universal Brønsted-Evans-Polanyi relations for various reaction classes [69], semi-empirical rate constant assessment and refinement [49,70], and group additivity and linear scaling methods[49] may also be 
utilized for reducing parameter uncertainty. The mechanism predictions are analyzed using spatial profiles of species mole/mass fractions and coverages, sensitivity analysis (SA), reaction path analysis (RPA), and reaction rate ratios, to identify the most important reaction pathways and kinetic parameters that control the model responses. Few kinetic parameters are then adjusted to improve the agreement with the experimental data. Further validation of the reaction mechanism is also carried out without adjusting any kinetic parameters. Next, we describe the application of this approach to ethanol reforming on Pt.

\subsection{Network of Species and Elementary Surface Reactions}

We have considered the following 16 species in our surface reaction mechanism: $\mathrm{C}_{2} \mathrm{H}_{5} \mathrm{OH}$ (ethanol), $\mathrm{C}_{2} \mathrm{H}_{5} \mathrm{O}$ (ethoxy), $\mathrm{CH}_{3} \mathrm{CHO}$ (acetaldehyde), $\mathrm{CH}_{3} \mathrm{CO}$ (acetyl), $\mathrm{CHCH}_{3}$ (ethylidene), $\mathrm{C}_{2} \mathrm{H}_{4}$ (ethylene), $\mathrm{CH}_{3}, \mathrm{CH}_{2}, \mathrm{CH}, \mathrm{C}, \mathrm{CO}, \mathrm{CO}_{2}, \mathrm{O}, \mathrm{H}, \mathrm{OH}$, and $\mathrm{H}_{2} \mathrm{O}$. In the proposed surface reaction mechanism, we have considered various adsorption/desorption steps along with the steps for thermal dehydrogenation, $\mathrm{O}^{*}$-assisted dehydrogenation, $\mathrm{OH}^{*}$ assisted dehydrogenation, C-C scission, and $\mathrm{C}-\mathrm{O}$ scission. Other reaction subsets such as, $\mathrm{CO}$ oxidation, $\mathrm{H}_{2}$ oxidation, and the coupling between the $\mathrm{CO}$ and $\mathrm{H}_{2}$ oxidation chemistries are considered as well. Reactions are chosen based on known reactants, products, and intermediates, as well as previously explored reaction pathways determined via first-principles calculations [50,53]. As non-elementary reactions and multiple competing pathways may be detrimental to the model performance [71], only elementary reactions are considered in this work. The mechanism primarily contains series, rather than parallel, reactions and only the sequential steps that are relevant to the species formation/destruction are considered to minimize the number of total reactions. Figure 3.2 shows an overall schematic of the C-containing species chemistry, exclusive of the side 
chemistries. The proposed mechanism consists of only 100 irreversible (50 reversible) elementary step reactions, as listed in Table $\mathbf{1 .}$

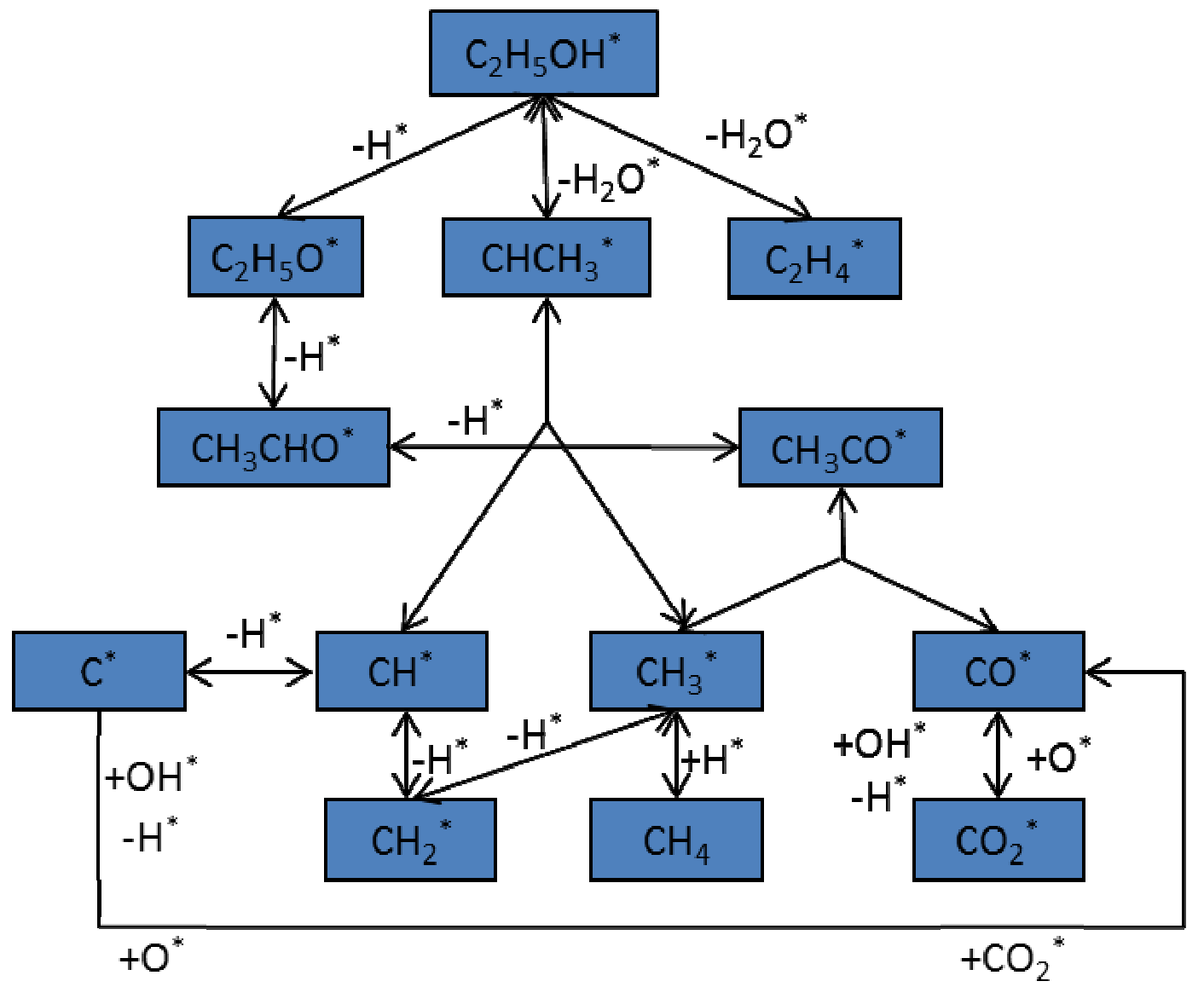

Figure 3.2: General Ethanol Reforming Reaction Mechanism 
Table 1: A 100-step surface reaction mechanism for ethanol reforming on Pt. Preexponential factor values in the parentheses correspond to the adjusted kinetic parameters in mechanism optimization.

\begin{tabular}{|c|c|c|c|c|}
\hline Number & Reaction & $\begin{array}{l}\text { Sticking } \\
\text { coefficient } \\
\text { s (-) or Pre- } \\
\text { exponential } \\
\text { factor A }\left(\mathrm{s}^{-1}\right)\end{array}$ & $\begin{array}{l}\text { Temperature } \\
\text { Exponent } \\
\beta(-)\end{array}$ & $\begin{array}{l}\text { Activation Energy } \\
\mathrm{E}(\mathrm{kcal} / \mathrm{mol}) \text { at } 300 \\
\mathrm{~K}\end{array}$ \\
\hline 1 & $\mathrm{O}_{2}+2^{*} \rightarrow 2 \mathrm{O}^{*}$ & $\mathrm{~s}=2.29 \times 10^{-2}$ & -0.05627 & 0.0 \\
\hline 2 & $2 \mathrm{O}^{*} \rightarrow \mathrm{O}_{2}+2^{*}$ & $4.37 \times 10^{12}$ & 0.05627 & $52.9-32 \theta_{\mathrm{O}}+f(\mathrm{~T})$ \\
\hline 3 & $\mathrm{O}+* \rightarrow \mathrm{O}^{*}$ & $\mathrm{~s}=3.16 \times 10^{-2}$ & -0.16990 & 0.0 \\
\hline 4 & $\mathrm{O}^{*} \rightarrow \mathrm{O}+*$ & $1.00 \times 10^{13}$ & 0.16990 & $86.0-16 \theta_{\mathrm{O}}+f(\mathrm{~T})$ \\
\hline 5 & $\mathrm{CO}+* \rightarrow \mathrm{CO}^{*}$ & $\mathrm{~s}=5.00 \times 10^{-1}$ & 0.16640 & 0.0 \\
\hline 6 & $\mathrm{CO}^{*} \rightarrow \mathrm{CO}+*$ & $7.11 \times 10^{13}$ & -0.16640 & $42.0-15 \theta_{\mathrm{CO}}+f(\mathrm{~T})$ \\
\hline 7 & $\mathrm{CO}_{2}+{ }^{*} \rightarrow \mathrm{CO}_{2}{ }^{*}$ & $\mathrm{~s}=2.89 \times 10^{-1}$ & -0.07339 & 0.0 \\
\hline 8 & $\mathrm{CO}_{2}{ }^{*} \rightarrow \mathrm{CO}_{2}{ }^{*}$ & $1.09 \times 10^{12}$ & 0.07339 & $4.5+f(\mathrm{~T})$ \\
\hline 9 & $\mathrm{H}_{2}+2^{*} \rightarrow 2 \mathrm{H}^{*}$ & $\begin{array}{l}\mathrm{s}=2.22 \times 10^{-2} \\
\left(2.22 \times 10^{-4}\right)\end{array}$ & -0.14198 & 0.0 \\
\hline 10 & $2 \mathrm{H}^{*} \rightarrow \mathrm{H}_{2}+2^{*}$ & $\begin{array}{l}1.43 \times 10^{13} \\
\left(1.43 \times 10^{11}\right)\end{array}$ & 0.14198 & $20.8-6 \theta_{\mathrm{H}}+f(\mathrm{~T})$ \\
\hline 11 & $\mathrm{H}+* \rightarrow \mathrm{H}^{*}$ & $\mathrm{~s}=2.90 \times 10^{-2}$ & -0.28732 & 0.0 \\
\hline 12 & $\mathrm{H}^{*} \rightarrow \mathrm{H}+*$ & $1.09 \times 10^{13}$ & 0.28732 & $62.5-3 \theta_{\mathrm{H}}+f(\mathrm{~T})$ \\
\hline 13 & $\mathrm{OH}+* \rightarrow \mathrm{OH}^{*}$ & $\mathrm{~s}=1.34 \times 10^{-2}$ & -0.05663 & 0.0 \\
\hline 14 & $\mathrm{OH}^{*} \rightarrow \mathrm{OH}+*$ & $2.36 \times 10^{13}$ & 0.05663 & $60.0-33 \theta_{\mathrm{O}}+f(\mathrm{~T})$ \\
\hline
\end{tabular}




\begin{tabular}{|c|c|c|c|c|}
\hline 15 & $\mathrm{H}_{2} \mathrm{O}+* \rightarrow \mathrm{H}_{2} \mathrm{O}^{*}$ & $\mathrm{~s}=1.52 \times 10^{-2}$ & 0.12717 & 0.0 \\
\hline 16 & $\mathrm{H}_{2} \mathrm{O}^{*} \rightarrow \mathrm{H}_{2} \mathrm{O}+*$ & $2.08 \times 10^{13}$ & -0.12717 & $10.2+f(\mathrm{~T})$ \\
\hline 17 & $\mathrm{C}+* \rightarrow \mathrm{C}^{*}$ & $\mathrm{~s}=3.33 \times 10^{-2}$ & -0.00417 & 0.0 \\
\hline 18 & $\mathrm{C}^{*} \rightarrow \mathrm{C}+*$ & $9.48 \times 10^{12}$ & 0.00417 & $162.6+f(\mathrm{~T})$ \\
\hline 19 & $\mathrm{CH}+* \rightarrow \mathrm{CH}^{*}$ & $\mathrm{~s}=1.36 \times 10^{-2}$ & 0.04146 & 0.0 \\
\hline 20 & $\mathrm{CH}^{*} \rightarrow \mathrm{CH}+*$ & $2.32 \times 10^{13}$ & -0.04146 & $163.3+f(\mathrm{~T})$ \\
\hline 21 & $\mathrm{CH}_{2}+* \rightarrow \mathrm{CH}_{2}^{*}$ & $\mathrm{~s}=1.20 \times 10^{-2}$ & 0.09401 & 0.0 \\
\hline 22 & $\mathrm{CH}_{2}{ }^{*} \rightarrow \mathrm{CH}_{2}+*$ & $2.64 \times 10^{13}$ & -0.09401 & $95.0+f(\mathrm{~T})$ \\
\hline 23 & $\mathrm{CH}_{3}+{ }^{*} \rightarrow \mathrm{CH}_{3}{ }^{*}$ & $\mathrm{~s}=1.92 \times 10^{-2}$ & 0.02960 & 0.0 \\
\hline 24 & $\mathrm{CH}_{3}{ }^{*} \rightarrow \mathrm{CH}_{3}+*$ & $1.65 \times 10^{13}$ & -0.02960 & $49.8+f(\mathrm{~T})$ \\
\hline 25 & $\mathrm{CH}_{4}+2^{*} \rightarrow \mathrm{CH}_{3}{ }^{*}+\mathrm{H}^{*}$ & $\begin{array}{l}s=5.96 \times 10^{-2} \\
\left(4.77 \times 10^{-3}\right)\end{array}$ & 0.15146 & $7.2+f\left(\mathrm{~T}, \theta_{\mathrm{H}}\right)$ \\
\hline 26 & $\mathrm{CH}_{3}{ }^{*}+\mathrm{H}^{*} \rightarrow \mathrm{CH}_{4}+2^{*}$ & $\begin{array}{l}5.31 \times 10^{12} \\
\left(4.25 \times 10^{11}\right)\end{array}$ & -0.15146 & $14.5+f\left(\mathrm{~T}, \theta_{\mathrm{H}}\right)$ \\
\hline 27 & $\mathrm{C}_{2} \mathrm{H}_{4}+* \rightarrow \mathrm{C}_{2} \mathrm{H}_{4}^{*}$ & $\mathrm{~s}=4.62 \times 10^{-2}$ & 0.10634 & 0.0 \\
\hline 28 & $\mathrm{C}_{2} \mathrm{H}_{4}{ }^{*} \rightarrow \mathrm{C}_{2} \mathrm{H}_{4}+*$ & $6.84 \times 10^{12}$ & -0.10634 & $17.5+f(\mathrm{~T})$ \\
\hline 29 & $\mathrm{CHCH}_{3}+* \rightarrow \mathrm{CHCH}_{3}^{*}$ & $\mathrm{~s}=4.37 \times 10^{-2}$ & -0.02472 & 0.0 \\
\hline 30 & $\mathrm{CHCH}_{3}{ }^{*} \rightarrow \mathrm{CHCH}_{3}+*$ & $7.24 \times 10^{12}$ & 0.02472 & $102.0+f(\mathrm{~T})$ \\
\hline 31 & $\mathrm{C}_{2} \mathrm{H}_{5} \mathrm{OH}+* \rightarrow \mathrm{C}_{2} \mathrm{H}_{5} \mathrm{OH}^{*}$ & $\mathrm{~s}=4.58 \times 10^{-2}$ & -0.06642 & 0.0 \\
\hline 32 & $\mathrm{C}_{2} \mathrm{H}_{5} \mathrm{OH}^{*} \rightarrow \mathrm{C}_{2} \mathrm{H}_{5} \mathrm{OH}+*$ & $6.90 \times 10^{12}$ & 0.06642 & $9.2+f(\mathrm{~T})$ \\
\hline 33 & $\mathrm{C}_{2} \mathrm{H}_{5} \mathrm{O}+* \rightarrow \mathrm{C}_{2} \mathrm{H}_{5} \mathrm{O}^{*}$ & $\mathrm{~s}=8.25 \times 10^{-2}$ & -0.06995 & 0.0 \\
\hline 34 & $\mathrm{C}_{2} \mathrm{H}_{5} \mathrm{O}^{*} \rightarrow \mathrm{C}_{2} \mathrm{H}_{5} \mathrm{O}+*$ & $3.83 \times 10^{12}$ & 0.06995 & $36.1+f(\mathrm{~T})$ \\
\hline 35 & $\mathrm{CH}_{3} \mathrm{CHO}+* \rightarrow \mathrm{CH}_{3} \mathrm{CHO}^{*}$ & $\mathrm{~s}=5.32 \times 10^{-2}$ & 0.03235 & 0.0 \\
\hline 36 & $\mathrm{CH}_{3} \mathrm{CHO}^{*} \rightarrow \mathrm{CH}_{3} \mathrm{CHO}+*$ & $5.94 \times 10^{12}$ & -0.03235 & $11.2+f(\mathrm{~T})$ \\
\hline 37 & $\mathrm{CH}_{3} \mathrm{CO}+* \rightarrow \mathrm{CH}_{3} \mathrm{CO}^{*}$ & $\mathrm{~s}=3.87 \times 10^{-2}$ & 0.11413 & 0.0 \\
\hline
\end{tabular}




\begin{tabular}{|c|c|c|c|c|}
\hline 38 & $\mathrm{CH}_{3} \mathrm{CO}^{*} \rightarrow \mathrm{CH}_{3} \mathrm{CO}+*$ & $8.18 \times 10^{12}$ & -0.11413 & $57.8+f(\mathrm{~T})$ \\
\hline 39 & $\mathrm{CO}^{*}+* \rightarrow \mathrm{C}^{*}+\mathrm{O}^{*}$ & $1.23 \times 10^{11}$ & 0.00726 & $53.4+f\left(\mathrm{~T}, \theta_{\mathrm{O}}, \theta_{\mathrm{CO}}\right)$ \\
\hline 40 & $\mathrm{C}^{*}+\mathrm{O}^{*} \rightarrow \mathrm{CO}^{*}+*$ & $8.12 \times 10^{10}$ & -0.00726 & $2.8+f\left(\mathrm{~T}, \theta_{\mathrm{O}}, \theta_{\mathrm{CO}}\right)$ \\
\hline 41 & $\mathrm{CO}_{2}{ }^{*}+* \rightarrow \mathrm{CO}^{*}+\mathrm{O}^{*}$ & $\begin{array}{l}1.10 \times 10^{11} \\
\left(1.10 \times 10^{10}\right)\end{array}$ & -0.00225 & $16.0+f\left(\mathrm{~T}, \theta_{\mathrm{O}}, \theta_{\mathrm{CO}}\right)$ \\
\hline 42 & $\mathrm{CO}^{*}+\mathrm{O}^{*} \rightarrow \mathrm{CO}_{2}^{*}+*$ & $\begin{array}{l}9.07 \times 10^{10} \\
\left(9.07 \times 10^{9}\right)\end{array}$ & 0.00225 & $12.3+f\left(\mathrm{~T}, \theta_{\mathrm{O}}, \theta_{\mathrm{CO}}\right)$ \\
\hline 43 & $2 \mathrm{CO}^{*} \rightarrow \mathrm{C}^{*}+\mathrm{CO}_{2}{ }^{*}$ & $1.09 \times 10^{11}$ & 0.03277 & $46.9+f\left(\mathrm{~T}, \theta_{\mathrm{CO}}\right)$ \\
\hline 44 & $\mathrm{C}^{*}+\mathrm{CO}_{2}^{*} \rightarrow 2 \mathrm{CO}^{*}$ & $9.21 \times 10^{10}$ & -0.03277 & $0.0+f\left(\mathrm{~T}, \theta_{\mathrm{CO}}\right)$ \\
\hline 45 & $\mathrm{OH}^{*}+* \rightarrow \mathrm{H}^{*}+\mathrm{O}^{*}$ & $\begin{array}{l}1.03 \times 10^{11} \\
\left(5.16 \times 10^{12}\right)\end{array}$ & 0.02585 & $25.0+f\left(\mathrm{~T}, \theta_{\mathrm{O}}, \theta_{\mathrm{H}}\right)$ \\
\hline 46 & $\mathrm{H}^{*}+\mathrm{O}^{*} \rightarrow \mathrm{OH}^{*}+*$ & $\begin{array}{l}9.69 \times 10^{10} \\
\left(4.84 \times 10^{12}\right)\end{array}$ & -0.02585 & $11.2+f\left(\mathrm{~T}, \theta_{\mathrm{O}}, \theta_{\mathrm{H}}\right)$ \\
\hline 47 & $\mathrm{H}_{2} \mathrm{O}^{*}+* \rightarrow \mathrm{H}^{*}+\mathrm{OH}^{*}$ & $1.15 \times 10^{11}$ & -0.01035 & $18.8+f\left(\mathrm{~T}, \theta_{\mathrm{O}}, \theta_{\mathrm{H}}\right)$ \\
\hline 48 & $\mathrm{H}^{*}+\mathrm{OH}^{*} \rightarrow \mathrm{H}_{2} \mathrm{O}^{*}+*$ & $8.67 \times 10^{10}$ & 0.01035 & $11.8+f\left(\mathrm{~T}, \theta_{\mathrm{O}}, \theta_{\mathrm{H}}\right)$ \\
\hline 49 & $\mathrm{H}_{2} \mathrm{O}^{*}+\mathrm{O}^{*} \rightarrow 2 \mathrm{OH}^{*}$ & $1.12 \times 10^{11}$ & -0.04019 & $11.6+f\left(\mathrm{~T}, \theta_{\mathrm{O}}\right)$ \\
\hline 50 & $2 \mathrm{OH}^{*} \rightarrow \mathrm{H}_{2} \mathrm{O}^{*}+\mathrm{O}^{*}$ & $8.93 \times 10^{10}$ & 0.04019 & $18.4+f\left(\mathrm{~T}, \theta_{\mathrm{O}}\right)$ \\
\hline 51 & $\mathrm{CO}_{2}{ }^{*}+\mathrm{H}^{*} \rightarrow \mathrm{CO}^{*}+\mathrm{OH}^{*}$ & $1.08 \times 10^{11}$ & -0.04181 & $7.3+f\left(\mathrm{~T}, \theta_{\mathrm{O}}, \theta_{\mathrm{H}}, \theta_{\mathrm{CO}}\right)$ \\
\hline 52 & $\mathrm{CO}^{*}+\mathrm{OH}^{*} \rightarrow \mathrm{CO}_{2}{ }^{*}+\mathrm{H}^{*}$ & $9.22 \times 10^{10}$ & 0.04181 & $17.4+f\left(\mathrm{~T}, \theta_{\mathrm{O}}, \theta_{\mathrm{H}}, \theta_{\mathrm{CO}}\right)$ \\
\hline 53 & $\mathrm{CO}^{*}+\mathrm{H}^{*} \rightarrow \mathrm{CH}^{*}+\mathrm{O}^{*}$ & $1.28 \times 10^{11}$ & -0.00285 & $44.1+f\left(\mathrm{~T}, \theta_{\mathrm{O}}, \theta_{\mathrm{H}}, \theta_{\mathrm{CO}}\right)$ \\
\hline 54 & $\mathrm{CH}^{*}+\mathrm{O}^{*} \rightarrow \mathrm{CO}^{*}+\mathrm{H}^{*}$ & $7.79 \times 10^{10}$ & 0.00285 & $12.3+f\left(\mathrm{~T}, \theta_{\mathrm{O}}, \theta_{\mathrm{H}}, \theta_{\mathrm{CO}}\right)$ \\
\hline 55 & $\mathrm{CO}^{*}+\mathrm{H}^{*} \rightarrow \mathrm{C}^{*}+\mathrm{OH}^{*}$ & $1.20 \times 10^{11}$ & -0.02030 & $40.4+f\left(\mathrm{~T}, \theta_{\mathrm{O}}, \theta_{\mathrm{H}}, \theta_{\mathrm{CO}}\right)$ \\
\hline 56 & $\mathrm{C}^{*}+\mathrm{OH}^{*} \rightarrow \mathrm{CO}^{*}+\mathrm{H}^{*}$ & $8.36 \times 10^{10}$ & 0.02030 & $3.5+f\left(\mathrm{~T}, \theta_{\mathrm{O}}, \theta_{\mathrm{H}}, \theta_{\mathrm{CO}}\right)$ \\
\hline 57 & $\mathrm{CH}^{*}+* \rightarrow \mathrm{C}^{*}+\mathrm{H}^{*}$ & $9.56 \times 10^{10}$ & 0.01239 & $32.0+f\left(\mathrm{~T}, \theta_{\mathrm{H}}\right)$ \\
\hline 58 & $\mathrm{C}^{*}+\mathrm{H}^{*} \rightarrow \mathrm{CH}^{*}+*$ & $1.05 \times 10^{11}$ & -0.01239 & $13.2+f\left(\mathrm{~T}, \theta_{\mathrm{H}}\right)$ \\
\hline
\end{tabular}




\begin{tabular}{|c|c|c|c|c|}
\hline 59 & $\mathrm{CH}_{2}^{*}+* \rightarrow \mathrm{CH}^{*}+\mathrm{H}^{*}$ & $1.01 \times 10^{11}$ & 0.02257 & $7.8+f\left(\mathrm{~T}, \theta_{\mathrm{H}}\right)$ \\
\hline 60 & $\mathrm{CH}^{*}+\mathrm{H}^{*} \rightarrow \mathrm{CH}_{2}^{*}+*$ & $9.94 \times 10^{10}$ & -0.02257 & $37.4+f\left(\mathrm{~T}, \theta_{\mathrm{H}}\right)$ \\
\hline 61 & $\mathrm{CH}_{3}{ }^{*}+* \rightarrow \mathrm{CH}_{2}^{*}+\mathrm{H}^{*}$ & $\begin{array}{l}1.17 \times 10^{11} \\
\left(1.17 \times 10^{10}\right)\end{array}$ & 0.08395 & $20.4+f\left(\mathrm{~T}, \theta_{\mathrm{H}}\right)$ \\
\hline 62 & $\mathrm{CH}_{2}{ }^{*}+\mathrm{H}^{*} \rightarrow \mathrm{CH}_{3}{ }^{*}+*$ & $\begin{array}{l}8.52 \times 10^{10} \\
\left(8.52 \times 10^{9}\right)\end{array}$ & -0.08395 & $17.3+f\left(\mathrm{~T}, \theta_{\mathrm{H}}\right)$ \\
\hline 63 & $\mathrm{C}^{*}+\mathrm{H}_{2} \mathrm{O}^{*} \rightarrow \mathrm{CH}^{*}+\mathrm{OH}^{*}$ & $1.21 \times 10^{11}$ & -0.02281 & $16.0+f\left(\mathrm{~T}, \theta_{\mathrm{O}}\right)$ \\
\hline 64 & $\mathrm{CH}^{*}+\mathrm{OH}^{*} \rightarrow \mathrm{C}^{*}+\mathrm{H}_{2} \mathrm{O}^{*}$ & $8.29 \times 10^{10}$ & 0.02281 & $27.8+f\left(\mathrm{~T}, \theta_{\mathrm{O}}\right)$ \\
\hline 65 & $\mathrm{C}^{*}+\mathrm{OH}^{*} \rightarrow \mathrm{CH}^{*}+\mathrm{O}^{*}$ & $1.09 \times 10^{11}$ & 0.00106 & $25.6+f\left(\mathrm{~T}, \theta_{\mathrm{O}}\right)$ \\
\hline 66 & $\mathrm{CH}^{*}+\mathrm{O}^{*} \rightarrow \mathrm{C}^{*}+\mathrm{OH}^{*}$ & $9.20 \times 10^{10}$ & -0.00106 & $30.7+f\left(\mathrm{~T}, \theta_{\mathrm{O}}\right)$ \\
\hline 67 & $\mathrm{CH}^{*}+\mathrm{H}_{2} \mathrm{O}^{*} \rightarrow \mathrm{CH}_{2}{ }^{*}+\mathrm{OH}^{*}$ & $1.14 \times 10^{11}$ & -0.03068 & $36.7+f\left(\mathrm{~T}, \theta_{\mathrm{O}}\right)$ \\
\hline 68 & $\mathrm{CH}_{2}{ }^{*}+\mathrm{OH}^{*} \rightarrow \mathrm{CH}^{*}+\mathrm{H}_{2} \mathrm{O}^{*}$ & $8.77 \times 10^{10}$ & 0.03068 & $0.0+f\left(\mathrm{~T}, \theta_{\mathrm{O}}\right)$ \\
\hline 69 & $\mathrm{CH}^{*}+\mathrm{OH}^{*} \rightarrow \mathrm{CH}_{2}{ }^{*}+\mathrm{O}^{*}$ & $1.02 \times 10^{11}$ & 0.00651 & $44.3+f\left(\mathrm{~T}, \theta_{\mathrm{O}}\right)$ \\
\hline 70 & $\mathrm{CH}_{2}{ }^{*}+\mathrm{O}^{*} \rightarrow \mathrm{CH}^{*}+\mathrm{OH}^{*}$ & $9.78 \times 10^{10}$ & -0.00651 & $0.9+f\left(\mathrm{~T}, \theta_{\mathrm{O}}\right)$ \\
\hline 71 & $\mathrm{CH}_{2}{ }^{*}+\mathrm{H}_{2} \mathrm{O}^{*} \rightarrow \mathrm{CH}_{3}{ }^{*}+\mathrm{OH}^{*}$ & $9.37 \times 10^{10}$ & -0.05611 & $15.6+f\left(\mathrm{~T}, \theta_{\mathrm{O}}\right)$ \\
\hline 72 & $\mathrm{CH}_{3}{ }^{*}+\mathrm{OH}^{*} \rightarrow \mathrm{CH}_{2}{ }^{*}+\mathrm{H}_{2} \mathrm{O}^{*}$ & $1.07 \times 10^{11}$ & 0.05611 & $11.6+f\left(\mathrm{~T}, \theta_{\mathrm{O}}\right)$ \\
\hline 73 & $\mathrm{CH}_{3}{ }^{*}+\mathrm{O}^{*} \rightarrow \mathrm{CH}_{2}{ }^{*}+\mathrm{OH}^{*}$ & $1.15 \times 10^{11}$ & 0.04137 & $13.1+f\left(\mathrm{~T}, \theta_{\mathrm{O}}\right)$ \\
\hline 74 & $\mathrm{CH}_{2}{ }^{*}+\mathrm{OH}^{*} \rightarrow \mathrm{CH}_{3}{ }^{*}+\mathrm{O}^{*}$ & $8.67 \times 10^{10}$ & -0.04137 & $23.7+f\left(\mathrm{~T}, \theta_{\mathrm{O}}\right)$ \\
\hline 75 & $\mathrm{CHCH}_{3}{ }^{*}+* \rightarrow \mathrm{CH}_{3}{ }^{*}+\mathrm{CH}^{*}$ & $1.25 \times 10^{11}$ & -0.06387 & $9.1+f(\mathrm{~T})$ \\
\hline 76 & $\mathrm{CH}_{3}{ }^{*}+\mathrm{CH}^{*} \rightarrow \mathrm{CHCH}_{3}{ }^{*}+*$ & $8.02 \times 10^{10}$ & 0.06387 & $29.0+f(\mathrm{~T})$ \\
\hline 77 & $\mathrm{C}_{2} \mathrm{H}_{5} \mathrm{O}^{*}+\mathrm{H}_{2} \mathrm{O}^{*} \rightarrow \mathrm{C}_{2} \mathrm{H}_{5} \mathrm{OH}^{*}+\mathrm{OH}^{*}$ & $1.22 \times 10^{11}$ & -0.00714 & $0.1+f\left(\mathrm{~T}, \theta_{\mathrm{O}}\right)$ \\
\hline 78 & $\mathrm{C}_{2} \mathrm{H}_{5} \mathrm{OH}^{*}+\mathrm{OH}^{*} \rightarrow \mathrm{C}_{2} \mathrm{H}_{5} \mathrm{O}^{*}+\mathrm{H}_{2} \mathrm{O}^{*}$ & $8.17 \times 10^{10}$ & 0.00714 & $7.9+f\left(\mathrm{~T}, \theta_{\mathrm{O}}\right)$ \\
\hline 79 & $\mathrm{C}_{2} \mathrm{H}_{5} \mathrm{OH}^{*}+\mathrm{O}^{*} \rightarrow \mathrm{C}_{2} \mathrm{H}_{5} \mathrm{O}^{*}+\mathrm{OH}^{*}$ & $9.58 \times 10^{10}$ & -0.04738 & $11.8+f\left(\mathrm{~T}, \theta_{\mathrm{O}}\right)$ \\
\hline 80 & $\mathrm{C}_{2} \mathrm{H}_{5} \mathrm{O}^{*}+\mathrm{OH}^{*} \rightarrow \mathrm{C}_{2} \mathrm{H}_{5} \mathrm{OH}^{*}+\mathrm{O}^{*}$ & $1.04 \times 10^{11}$ & 0.04738 & $10.8+f\left(\mathrm{~T}, \theta_{\mathrm{O}}\right)$ \\
\hline 81 & $\mathrm{C}_{2} \mathrm{H}_{5} \mathrm{OH}^{*}+* \rightarrow \mathrm{C}_{2} \mathrm{H}_{5} \mathrm{O}^{*}+\mathrm{H}^{*}$ & $9.44 \times 10^{10}$ & -0.00527 & $18.8+f\left(\mathrm{~T}, \theta_{\mathrm{H}}\right)$ \\
\hline
\end{tabular}




\begin{tabular}{|c|c|c|c|c|}
\hline 82 & $\mathrm{C}_{2} \mathrm{H}_{5} \mathrm{O}^{*}+\mathrm{H}^{*} \rightarrow \mathrm{C}_{2} \mathrm{H}_{5} \mathrm{OH}^{*}+*$ & $1.06 \times 10^{11}$ & 0.00527 & $4.1+f\left(\mathrm{~T}, \theta_{\mathrm{H}}\right)$ \\
\hline 83 & $\mathrm{C}_{2} \mathrm{H}_{5} \mathrm{OH}^{*}+* \rightarrow \mathrm{C}_{2} \mathrm{H}_{4}^{*}+\mathrm{H}_{2} \mathrm{O}^{*}$ & $\begin{array}{l}1.16 \times 10^{11} \\
\left(4.64 \times 10^{10}\right)\end{array}$ & -0.10634 & $0.0+f(\mathrm{~T})$ \\
\hline 84 & $\mathrm{C}_{2} \mathrm{H}_{4}{ }^{*}+\mathrm{H}_{2} \mathrm{O}^{*} \rightarrow \mathrm{C}_{2} \mathrm{H}_{5} \mathrm{OH}^{*}+*$ & $\begin{array}{l}8.62 \times 10^{10} \\
\left(3.45 \times 10^{10}\right)\end{array}$ & 0.10634 & $7.6+f(\mathrm{~T})$ \\
\hline 85 & $\mathrm{C}_{2} \mathrm{H}_{5} \mathrm{OH}^{*}+* \rightarrow \mathrm{CHCH}_{3}^{*}+\mathrm{H}_{2} \mathrm{O}^{*}$ & $\begin{array}{l}2.93 \times 10^{11} \\
\left(6.44 \times 10^{11}\right)\end{array}$ & -0.06413 & $0.0+f(\mathrm{~T})$ \\
\hline 86 & $\mathrm{CHCH}_{3}{ }^{*}+\mathrm{H}_{2} \mathrm{O}^{*} \rightarrow \mathrm{C}_{2} \mathrm{H}_{5} \mathrm{OH}^{*}+*$ & $\begin{array}{l}3.41 \times 10^{10} \\
\left(7.51 \times 10^{10}\right)\end{array}$ & 0.06413 & $17.9+f(\mathrm{~T})$ \\
\hline 87 & $\mathrm{CH}_{3} \mathrm{CHO}^{*}+\mathrm{H}_{2} \mathrm{O}^{*} \rightarrow \mathrm{C}_{2} \mathrm{H}_{5} \mathrm{O}^{*}+\mathrm{OH}^{*}$ & $9.00 \times 10^{10}$ & -0.07331 & $28.1+f\left(\mathrm{~T}, \theta_{\mathrm{O}}\right)$ \\
\hline 88 & $\mathrm{C}_{2} \mathrm{H}_{5} \mathrm{O}^{*}+\mathrm{OH}^{*} \rightarrow \mathrm{CH}_{3} \mathrm{CHO}^{*}+\mathrm{H}_{2} \mathrm{O}^{*}$ & $1.11 \times 10^{11}$ & 0.07331 & $0.0+f\left(\mathrm{~T}, \theta_{\mathrm{O}}\right)$ \\
\hline 89 & $\mathrm{CH}_{3} \mathrm{CHO}^{*}+\mathrm{OH}^{*} \rightarrow \mathrm{C}_{2} \mathrm{H}_{5} \mathrm{O}^{*}+\mathrm{O}^{*}$ & $7.12 \times 10^{10}$ & 0.04173 & $34.8+f\left(\mathrm{~T}, \theta_{\mathrm{O}}\right)$ \\
\hline 90 & $\mathrm{C}_{2} \mathrm{H}_{5} \mathrm{O}^{*}+\mathrm{O}^{*} \rightarrow \mathrm{CH}_{3} \mathrm{CHO}^{*}+\mathrm{OH}^{*}$ & $1.40 \times 10^{11}$ & -0.04173 & $0.0+f\left(\mathrm{~T}, \theta_{\mathrm{O}}\right)$ \\
\hline 91 & $\mathrm{C}_{2} \mathrm{H}_{5} \mathrm{O}^{*}+* \rightarrow \mathrm{CH}_{3} \mathrm{CHO}^{*}+\mathrm{H}^{*}$ & $1.43 \times 10^{11}$ & -0.00219 & $0.0+f\left(\mathrm{~T}, \theta_{\mathrm{H}}\right)$ \\
\hline 92 & $\mathrm{CH}_{3} \mathrm{CHO}^{*}+\mathrm{H}^{*} \rightarrow \mathrm{C}_{2} \mathrm{H}_{5} \mathrm{O}^{*}+*$ & $7.00 \times 10^{10}$ & 0.00219 & $21.1+f\left(\mathrm{~T}, \theta_{\mathrm{H}}\right)$ \\
\hline 93 & $\mathrm{CH}_{3} \mathrm{CO}^{*}+\mathrm{H}_{2} \mathrm{O}^{*} \rightarrow \mathrm{CH}_{3} \mathrm{CHO}^{*}+\mathrm{OH}^{*}$ & $6.93 \times 10^{10}$ & 0.01204 & $29.7+f\left(\mathrm{~T}, \theta_{\mathrm{O}}\right)$ \\
\hline 94 & $\mathrm{CH}_{3} \mathrm{CHO}^{*}+\mathrm{OH}^{*} \rightarrow \mathrm{CH}_{3} \mathrm{CO}^{*}+\mathrm{H}_{2} \mathrm{O}^{*}$ & $1.44 \times 10^{11}$ & -0.01204 & $0.0+f\left(\mathrm{~T}, \theta_{\mathrm{O}}\right)$ \\
\hline 95 & $\mathrm{CH}_{3} \mathrm{CO}^{*}+\mathrm{OH}^{*} \rightarrow \mathrm{CH}_{3} \mathrm{CHO}^{*}+\mathrm{O}^{*}$ & $6.46 \times 10^{10}$ & 0.02464 & $36.4+f\left(\mathrm{~T}, \theta_{\mathrm{O}}\right)$ \\
\hline 96 & $\mathrm{CH}_{3} \mathrm{CHO}^{*}+\mathrm{O}^{*} \rightarrow \mathrm{CH}_{3} \mathrm{CO}^{*}+\mathrm{OH}^{*}$ & $1.55 \times 10^{11}$ & -0.02464 & $0.0+f\left(\mathrm{~T}, \theta_{\mathrm{O}}\right)$ \\
\hline 97 & $\mathrm{CH}_{3} \mathrm{CHO}^{*}+* \rightarrow \mathrm{CH}_{3} \mathrm{CO}^{*}+\mathrm{H}^{*}$ & $1.57 \times 10^{11}$ & 0.02335 & $3.7+f\left(\mathrm{~T}, \theta_{\mathrm{H}}\right)$ \\
\hline 98 & $\mathrm{CH}_{3} \mathrm{CO}^{*}+\mathrm{H}^{*} \rightarrow \mathrm{CH}_{3} \mathrm{CHO}^{*}+*$ & $6.39 \times 10^{10}$ & -0.02335 & $26.4+f\left(\mathrm{~T}, \theta_{\mathrm{H}}\right)$ \\
\hline 99 & $\mathrm{CH}_{3} \mathrm{CO}^{*}+* \rightarrow \mathrm{CH}_{3}^{*}+\mathrm{CO}^{*}$ & $1.59 \times 10^{11}$ & -0.11616 & $1.4+f\left(\mathrm{~T}, \theta_{\mathrm{CO}}\right)$ \\
\hline 100 & $\mathrm{CH}_{3}{ }^{*}+\mathrm{CO}^{*} \rightarrow \mathrm{CH}_{3} \mathrm{CO}^{*}+*$ & $6.31 \times 10^{10}$ & 0.11616 & $21.3+f\left(\mathrm{~T}, \theta_{\mathrm{CO}}\right)$ \\
\hline
\end{tabular}




\subsection{Kinetic Parameter Estimation}

Each elementary reaction in the mechanism is associated with three kinetic parameters: (i) pre-exponential factor $\mathrm{A}$, or sticking coefficient $\mathrm{s}$, (ii) temperature exponent $\beta$, and (iii) activation energy E, which can be found for each reaction in Table 1. Sticking coefficients for the adsorption reactions are taken from values reported in literature, or otherwise taken as unity. Pre-exponential factors are taken from typical estimates based on Transition State Theory (e.g., $10^{13} \mathrm{~s}^{-1}$ for desorption and $10^{11} \mathrm{~s}^{-1}$ for Langmuir-Hinshelwood type surface reaction) [56]. Site density $\sigma$, is taken as $1.5 \times 10^{15}$ sites $/ \mathrm{cm}^{2}\left(2.5 \times 10^{-9} \mathrm{~mol} / \mathrm{cm}^{2}\right)$ based on the typical estimate for a $\operatorname{Pt}(111)$ facet. Species binding energies are based on experimental or DFT values reported in literature and are listed in Table 2. Binding energies are coverage $(\theta)$ and temperature (T) dependent, as shown in Eq. 1.

$$
\mathrm{Q}(\mathrm{T})=\mathrm{Q}\left(\mathrm{T}_{\mathrm{o}}\right)-\alpha \theta-\gamma \mathrm{R}\left(\mathrm{T}-\mathrm{T}_{\mathrm{o}}\right)
$$

Coverage dependence (adsorbate-adsorbate interactions) is taken from values reported in literature [72]. The temperature dependence from statistical mechanics is rooted in the degrees of freedom lost and gained by the species upon adsorption, and are treated as described in [67]. The coverage and temperature dependence coefficients ( $\alpha$ and $\gamma)$ are listed in Table 2. This results in coverage and temperature dependent activation energies, which are calculated on-the-fly using the semi-empirical Unity Bond Index-Quadratic Exponential Potential (UBI-QEP) theory [73], and are reported in Table 1 at $300 \mathrm{~K}$. Bond index values in the UBI-QEP formalism are taken as 0.5 [73]. 


\subsection{Thermodynamic Consistency}

Thermodynamic consistency is an often overlooked, but important aspect in mechanism development $[13,57,63,67]$. Especially at or near equilibrium conditions, this may result in the calculation of incorrect reaction rates. UBI-QEP formalism utilizes thermodynamic loops to determine the activation energies using binding energies and hence inherently provides enthalpic consistency at the reaction and overall mechanism level. However, such a reaction mechanism still violates entropic consistency, as pre-exponentials are taken from approximate transition state theory estimates without any thermodynamic constraints. We have ensured entropic consistency in our surface reaction mechanism, at both the reaction and overall mechanism level, through the temperature exponent, $\beta$, in the modified Arrhenius equation [67]. Eq. 2 shows the relationship between the temperature dependent entropy and the modified pre-exponential factor. Here, $\mathrm{T}_{\mathrm{o}}$ is taken as $300 \mathrm{~K}$.

$$
\frac{\Delta S}{R}=\ln \left(\frac{A_{f}}{A_{b}}\right)+\left(\beta_{f}-\beta_{b}\right) \ln \left(\frac{T}{T_{o}}\right)
$$

In the same manner as [67], we have optimized the $\beta$ values and the $A_{f} / A_{b}$ ratios to ensure entropic consistency over a large temperature range of interest (300-2100 K). It is important to note that this parameter optimization is not against any particular experimental data set, but rather is to ensure that Hess's law is satisfied over a large temperature range. Figure 3.3 shows the ratios of equilibrium constants calculated using gas phase parameters vs. those using the surface reaction parameters. This ratio must be close to unity for a thermodynamically consistent surface reaction mechanism, thereby satisfying Hess's law. On the other hand, reactions in the thermodynamically inconsistent mechanism show a large disparity (e.g., as high as three orders of magnitude) in the 
equilibrium constant values. Kinetic parameters (A and $\beta$ ) for the thermodynamically consistent reaction mechanism are listed in Table 2.

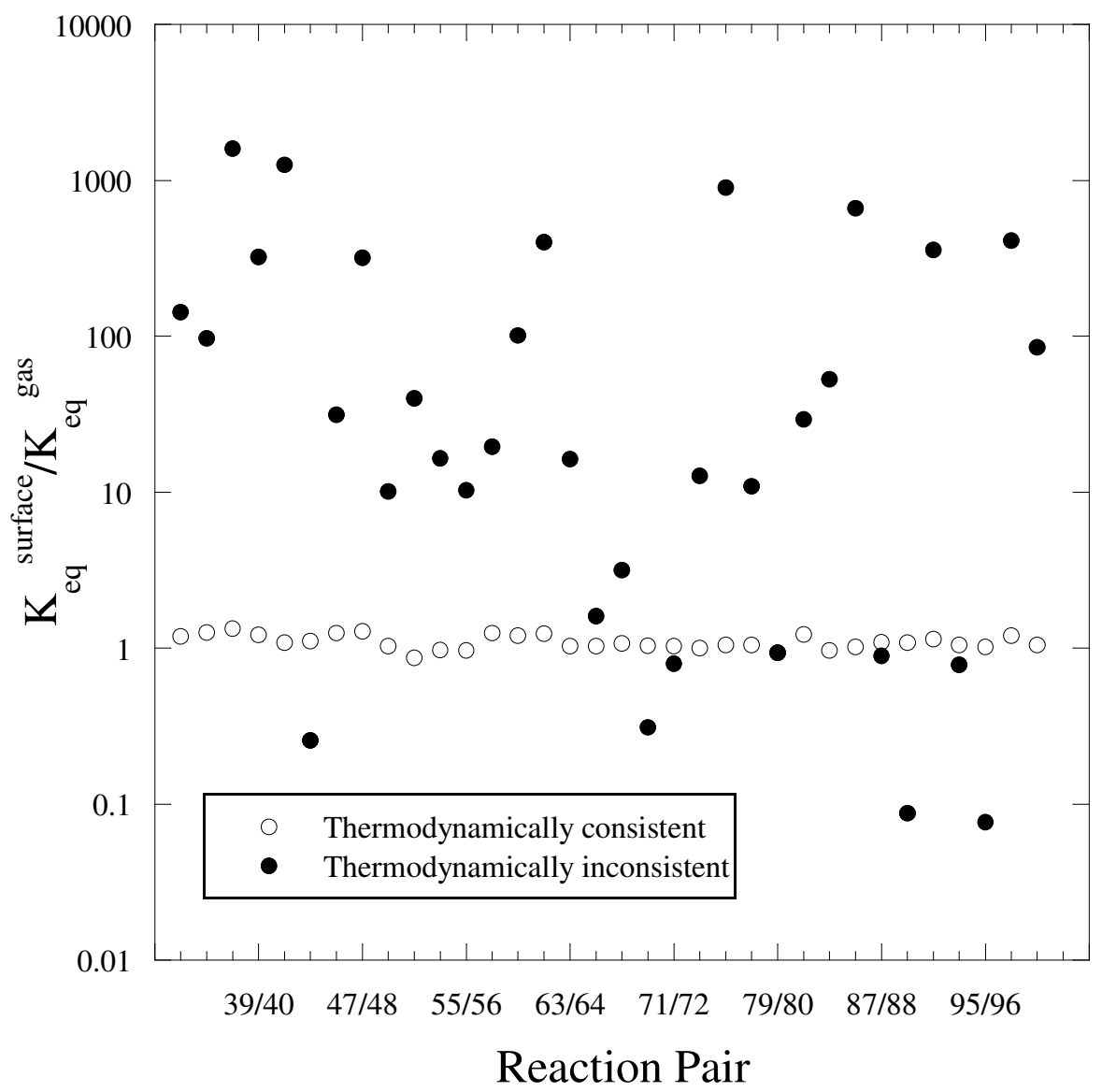

Figure 3.3.: $\mathrm{K}_{\mathrm{eq}}$ ratio $\left(\mathrm{K}_{\mathrm{eq}}{ }^{\text {surface }} / \mathrm{K}_{\mathrm{eq}}{ }^{\mathrm{gas}}\right)$ for reactions in the thermodynamically consistent and thermodynamically inconsistent surface reaction mechanisms 
Table 2: Species Binding Energies, Coverage and Temperature Dependence Coefficients

\begin{tabular}{|l|l|l|l|}
\hline Species & $\begin{array}{l}\text { Chosen binding } \\
\text { energy Q (kcal/mol) } \\
\text { and selected } \\
\text { references }\end{array}$ & $\begin{array}{l}\text { Coverage } \\
\text { dependence } \alpha \theta \\
(\mathrm{kcal} / \mathrm{mol})\end{array}$ & $\begin{array}{l}\text { Temperature } \\
\text { dependence } \gamma \\
(-)\end{array}$ \\
\hline $\mathrm{O}^{*}$ & 86.0 DFT [66] & $16 \theta_{\mathrm{O}}[72]$ & 1.5 \\
\hline $\mathrm{H}^{*}$ & 62.5 DFT [74] & $3 \theta_{\mathrm{H}}[72]$ & 1.5 \\
\hline $\mathrm{OH}^{*}$ & 60.0 Experiments [75] & $33 \theta_{\mathrm{O}}[72]$ & 2.0 \\
\hline $\mathrm{H}_{2} \mathrm{O}^{*}$ & 10.2 DFT [76] & - & 2.5 \\
\hline $\mathrm{CO}^{*}$ & 42.0 DFT [74] & $15 \theta_{\mathrm{CO}}[72]$ & 2.0 \\
\hline $\mathrm{CO}_{2}{ }^{*}$ & 4.5 DFT [77] & - & 2.0 \\
\hline $\mathrm{C}^{*}$ & 162.6 DFT [78] & - & 1.5 \\
\hline $\mathrm{CH}^{*}$ & 163.3 DFT [79,80] & - & 2.0 \\
\hline $\mathrm{CH}_{2}{ }^{*}$ & 95.0 DFT [78] & - & 3.0 \\
\hline $\mathrm{CH}_{3}{ }^{*}$ & 49.8 DFT [78] & - & 3.0 \\
\hline $\mathrm{C}_{2} \mathrm{H}_{4}{ }^{*}$ & 17.5 Experiments [81] & - & 3.0 \\
\hline $\mathrm{CHCH}_{3}{ }^{*}$ & 102.0 DFT [63,82] & - & 3.0 \\
\hline $\mathrm{C}_{2} \mathrm{H}_{5} \mathrm{OH}{ }^{*}$ & 9.2 DFT [49] & - & 3.0 \\
\hline $\mathrm{C}_{2} \mathrm{H}_{5} \mathrm{O}^{*}$ & 36.1 DFT [49] & - & - \\
\hline $\mathrm{CH}_{3} \mathrm{CHO}^{*}$ & 11.2 DFT [49] & - & 3.0 \\
\hline $\mathrm{CH}_{3} \mathrm{CO}^{*}$ & 57.8 DFT [49] & - & \\
\hline
\end{tabular}




\subsection{Reactor Modeling}

Fixed bed and washcoated foam experiments are modeled as a steady state isothermal plug flow reactor. The governing equations are shown below.

$$
\begin{array}{ll}
\frac{\mathrm{dY} Y_{k}}{d z}=\frac{G_{k} M_{k}}{\rho u}+\left(\frac{A_{c}}{V_{R}}\right) \frac{S_{k} M_{k}}{\rho u}, & \mathrm{k}=\text { gas species } \\
S_{k}=0, & \mathrm{k}=\text { surface species excluding empty sites } \\
\sum \theta_{\mathrm{k}}=1, & \mathrm{k}=\text { surface species including empty sites }
\end{array}
$$

As the surface species coverages at the reactor inlet are unknown, they are estimated using a transient simulation at the inlet, until steady state is reached. Subsequently, the set of differential-algebraic equations (DAEs) is solved using the DDASPK solver [83]. Mass transfer limitations, the importance of which has been highlighted by [84], are considered throughout the reactor length, even though mass transfer was found to be significant only in the entrance region of the reactor.

\subsection{Model Assumptions and Limitations}

Prior to discussing the results from this work, here we acknowledge the major assumptions/limitations related to the overall modeling approach.

\section{- Parameter uncertainty:}

- Uncertainty in the kinetic parameters arises from the use of various estimation methods and their accuracy. Species binding energies extracted from experiments vs. estimated from first-principles calculations vary significantly in some cases.

- As DFT-based activation energies are not available for all elementary reactions, they are calculated using the semi-empirical UBI-QEP method. Even though 
the UBI-QEP method is highly convenient in accounting for coverage effects and ensuring enthalpic consistency, the calculated activation energies need to be verified with first-principles DFT calculations.

○ Pt site density of $1.5 \times 10^{15}$ sites $/ \mathrm{cm}^{2}$ is calculated from one facet (111) of the Pt crystal, whereas the actually utilized catalysts are polycrystalline in nature.

○ Through the adjustment of kinetic parameters, uncertainty in the experimental measurements (if any) may be translated to the surface reaction mechanism.

\section{- Mechanistic limitations:}

- Coking and regeneration kinetics are not a significant issue on $\mathrm{Pt}$ and are not included in the surface reaction mechanism, but these chemistries will be important for other catalysts, such as $\mathrm{Ni}$ and Co.

○ The surface reaction mechanism does not include propanol and methanol species that are present, albeit in small amounts, in bio-ethanol derived from biomass sources.

$\circ$ The mechanism developed in this work is not exhaustive, and does not consider all possible species and reactions in the interest of practical implementation in reactor design. The omission of some reactions may result in cancellation of errors, which may lead to translation of uncertainty to the kinetic parameters.

\section{- Modeling limitations:}

- Given the uncertainty in the Marinov mechanism, as discussed earlier, a more reliable and rigorously validated gas phase reaction mechanism for $C_{2}$ and $C_{2+}$ oxygenate species needs to be developed. 
- As the temperature profile is not reported in the ethanol partial oxidation experimental data [22], the reactor modeling assumes isothermal operation at the reported temperature. Even though this may adequately represent the average reactor temperature, it is known that the typical partial oxidation reactors show a hot spot due to a combination of complete oxidation (exothermic) followed by reforming (endothermic).

○ The model performance for ethanol oxidation/reforming is limited by optimization against one set of experimental data [22], which may activate only a few dominant pathways. Monte Carlo-based methods have been used by Vlachos and colleagues to identify the operating conditions which may activate different types of pathways $[85,86]$. Along the same lines, additional methane dry reforming experiments were conducted (with different important pathways than the ethanol oxidation/reforming chemistry) to validate the surface reaction mechanism developed in this work. 


\section{CHAPTER 4: Performance and Optimization of the Surface Reaction Mechanism}

Reactor simulations were carried out to model the experimental data for partial oxidation of ethanol on Pt [22]. The experimental data reports ethanol conversion and product $\left(\mathrm{C}_{2} \mathrm{H}_{4}, \mathrm{CH}_{4}, \mathrm{CO}, \mathrm{H}_{2}\right.$, and $\left.\mathrm{H}_{2} \mathrm{O}\right)$ selectivities as a function of $\mathrm{C}: \mathrm{O}$ ratio, and corresponding reactor temperature, as shown in Figure 4.1. Figure 4.1A shows the model predictions using the thermodynamically consistent surface reaction mechanism of Table 2. The operating conditions are listed in the caption of Figure 4.1. The catalyst area per unit volume is calculated from the diameter of the pores in the foam $\left(\frac{A_{c}}{V_{R}}=\frac{4}{d}\right)$. The thermodynamically consistent reaction mechanism has superior predictions for $\mathrm{C}_{2} \mathrm{H}_{4}, \mathrm{CH}_{4}$, $\mathrm{CO}$, and $\mathrm{H}_{2} \mathrm{O}$ selectivities, compared to the initial thermodynamically inconsistent mechanism (not shown). However, the overall agreement with the experimental data is poor. Therefore, the mechanism predictions are analyzed using a variety of approaches including sensitivity analysis and spatial profile analysis for species coverages and mole fractions, as discussed next. 

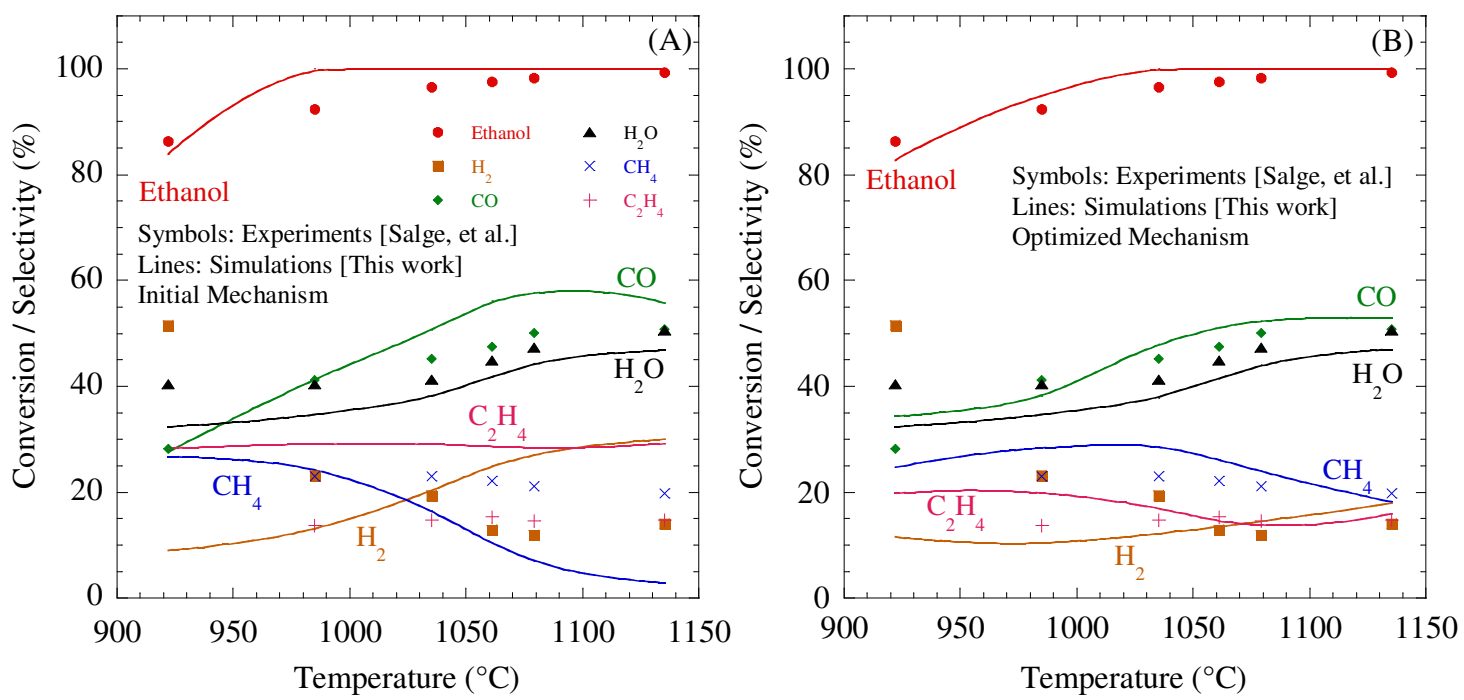

Figure 4.1 Comparison of the microkinetic model simulations from this work and the experimental data of [22]. Panels A and B represent the initial and optimized surface reaction mechanism, respectively. Symbols represent data from quartz tube reactor ethanol partial oxidation experiments. The operating conditions are as follows: inlet composition $=$ ethanol and air supplied at various $\mathrm{C}: \mathrm{O}$ ratios (and temperatures) of $1.1\left(922^{\circ} \mathrm{C}\right), 1.0(985$ $\left.{ }^{\circ} \mathrm{C}\right), 0.9\left(1035^{\circ} \mathrm{C}\right), 0.8\left(1061^{\circ} \mathrm{C}\right), 0.75\left(1079{ }^{\circ} \mathrm{C}\right)$, and $0.7\left(1135^{\circ} \mathrm{C}\right)$, pressure $=1 \mathrm{~atm}$, velocity $=49.1 \mathrm{~cm} / \mathrm{s}$, catalyst length $=1.0 \mathrm{~cm}$, and catalyst area per unit reactor volume $\mathrm{A}_{\mathrm{c}} / \mathrm{V}_{\mathrm{R}}=200 \mathrm{~cm}^{-1}$.

\subsection{Identification and Refinement of Important Parameters}

Sensitivity analysis was carried out with respect to the pre-exponential factors to identify the most influential reactions. The normalized sensitivity coefficient is defined as $\frac{d \ln R}{d \ln P}$, where $d \ln P$ is the change in parameter (pre-exponential factor) and $d \ln R$ is the change in model response (conversion or selectivity). As the pre-exponentials are already optimized to ensure entropic consistency, a pairwise sensitivity analysis was conducted [87] to maintain that consistency, i.e., pre-exponential factors of both the forward and backward reaction pair were perturbed by the same factor so that the equilibrium constant 
is not perturbed. A sample set of the highest sensitivity coefficients are shown in Figure 4.2. While choosing the magnitude of parameter adjustment for a particular model response, the simultaneous impact on the other model responses is considered as well, i.e., we attempt to change only those parameters, which have the highest sensitivity coefficient for a particular model response and a low sensitivity coefficient, and hence low impact, for the other model responses.

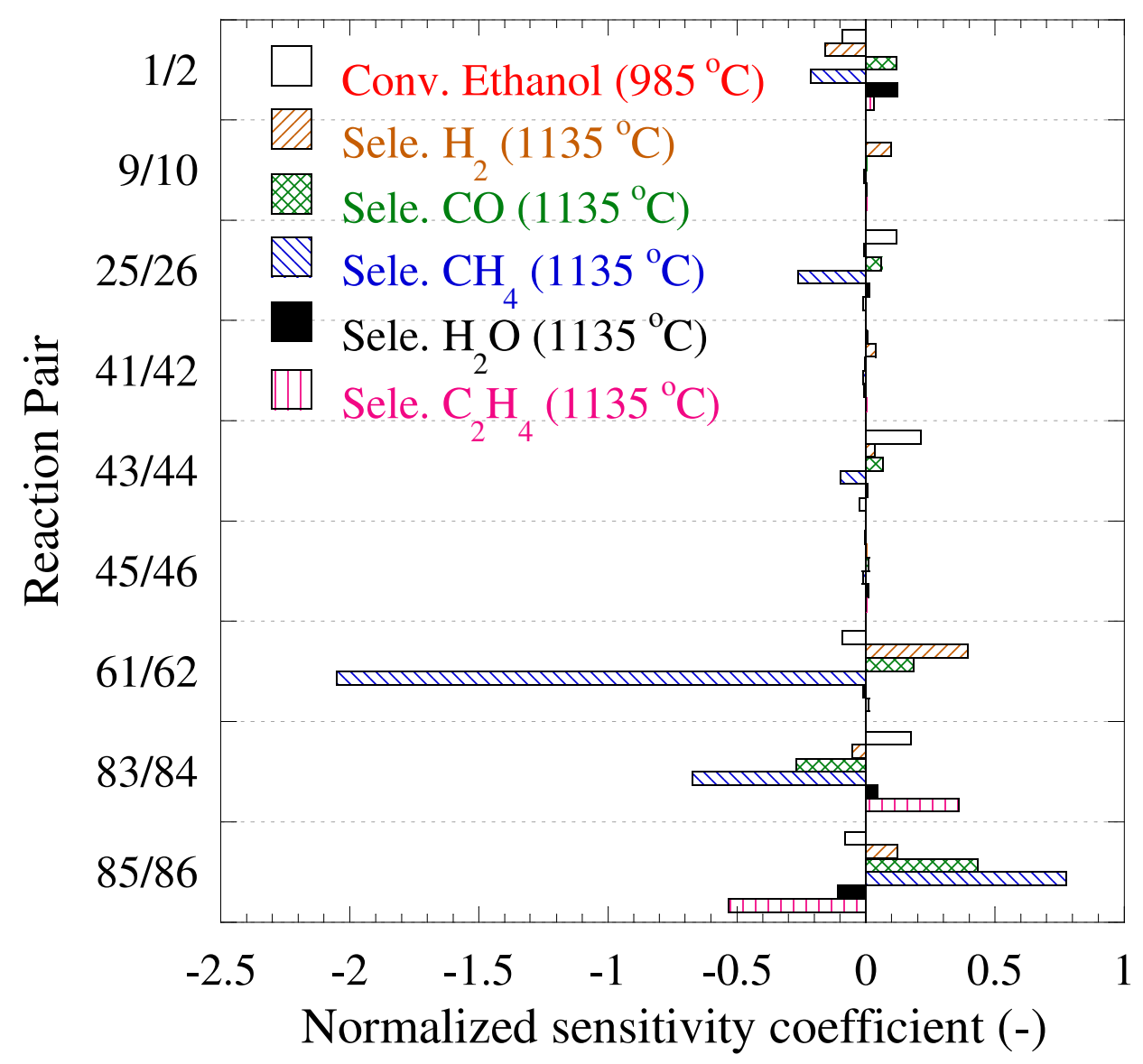

Figure 4.2: Sensitivity analysis for all model responses (ethanol conversion at $985^{\circ} \mathrm{C}$ and selectivities of $\mathrm{H}_{2}, \mathrm{CO}, \mathrm{CH}_{4}, \mathrm{H}_{2} \mathrm{O}$, and $\mathrm{C}_{2} \mathrm{H}_{4}$ at $1135^{\circ} \mathrm{C}$ ) with respect to the preexponential factors in the initial thermodynamically consistent surface reaction mechanism with a perturbation factor of 2. Operating conditions are the same as those in Figure 4.1. 
Based on this sensitivity analysis, only pre-exponential factors for seven surface reaction pairs were adjusted to improve the agreement with the experimental data. No bond indices or binding energies were adjusted. Although adjustment of the bond indices from the empirical value of 0.5 has recently been shown to improve the agreement between UBIQEP and DFT calculations [70], there is not enough information from DFT calculations to determine a more appropriate bond index value for each reaction in our surface reaction mechanism. As the initial estimates for pre-exponential factors may have an uncertainty off a factor of 10-100, we have chosen to optimize only the important preexponential factors.

The optimized parameters used in the final surface reaction mechanism are also listed in parentheses in Table 1. Reaction pair 9-10 $\left(\mathrm{H}_{2}\right.$ adsorption/desorption to/from $\left.2 \mathrm{H}^{*}\right)$ was adjusted to improve water selectivity predictions by keeping hydrogen on the surface and available to react with $\mathrm{OH}^{*}$. Reaction pair $25-26\left(\mathrm{CH}_{4}\right.$ adsorption/desorption to/from $\mathrm{CH}_{3}{ }^{*}+\mathrm{H}^{*}$ ) was tuned to decrease methane selectivity at low temperatures and increase it at higher temperatures. Reaction pair 41-42 $\left(\mathrm{CO}_{2}{ }^{*}\right.$ scission/formation to/from $\left.\mathrm{CO}^{*}+\mathrm{O}^{*}\right)$ controls CO selectivity, particularly at higher temperatures as the contribution of this reaction to $\mathrm{CO}_{2}{ }^{*}$ consumption increases with increasing temperature. Tuning of reaction pair 45-46 $\left(\mathrm{OH}^{*}\right.$ scission/formation to/from $\left.\mathrm{H}^{*}+\mathrm{O}^{*}\right)$ helped to improve water selectivity predictions. Reaction pair 61-62 $\left(\mathrm{CH}_{3}{ }^{*}\right.$ decomposition/formation to/from $\left.\mathrm{CH}_{2}{ }^{*}+\mathrm{H}^{*}\right)$ is important in controlling methane selectivity, as the alternate route for $\mathrm{CH}_{3}{ }^{*}$ consumption is to form methane. Finally, tuning of reaction pair 83-84 (ethanol dehydration/formation to/from $\mathrm{C}_{2} \mathrm{H}_{4}{ }^{*}+\mathrm{H}_{2} \mathrm{O}^{*}$ ) and reaction pair 85-86 (ethanol dehydration/formation to/from $\mathrm{CHCH}_{3}{ }^{*}+\mathrm{H}_{2} \mathrm{O}^{*}$ ) helped to control ethylene selectivity. 


\subsection{Mechanism Predictions against Experimental Data}

Figure 4.1B shows the predictions of the optimized surface reaction mechanism. The overall agreement with the experimental data is significantly improved, as compared to Figure 4.1A, especially for the $\mathrm{CO}, \mathrm{CH}_{4}$, and $\mathrm{C}_{2} \mathrm{H}_{4}$ selectivities. We note that there is a large discrepancy for the $\mathrm{H}_{2}$ selectivity predictions, especially at the lowest temperature of $922^{\circ} \mathrm{C}$. We believe that the $\mathrm{H}_{2}$ selectivity experimental data point at $922^{\circ} \mathrm{C}$ is a possible outlier for four reasons: (i) Salge et al. state that at low C:O ratios the mixture was unstable [22]. (ii) Product selectivities for methane and ethylene are not reported at this temperature [22], which further indicates the uncertainty in the measurements. (iii) The model predictions in Figure 4.1B are in good agreement with all the other reported selectivity values. (iv) Based on the model predicted trend for the $\mathrm{H}_{2}$ selectivity, it should be a low value at $922{ }^{\circ} \mathrm{C}$, which should be more difficult to measure. Nonetheless, it is important to note that the microkinetic model shows good agreement with the overall experimental data with the adjustment of a few kinetic parameters. To understand the fundamental chemistry on Pt under the operating conditions, next the findings from detailed mechanistic and reaction path analysis are discussed. 


\section{CHAPTER 5: Mechanistic Analysis and Reaction Zones}

Reaction Path Analysis (RPA) as well as analysis of coverage profiles, mole fraction profiles, and rate ratios, were used to investigate the dominant species and reactions as a function of the catalyst length. In RPA, the relative contribution of competing pathways in producing/consuming a selected species is computed. Repeating this for all species yields the network of the most dominant reactions. Reaction pathways were determined for both the gas and surface phases. Combination of RPA with coverage profiles, mole fraction profiles, and reaction rate ratios provides information about how the dominant chemistry varies over the catalyst length - potentially valuable information in reactor design/optimization. Axial profiles of gas species mole fractions at three temperatures $\left(922{ }^{\circ} \mathrm{C}, 1035{ }^{\circ} \mathrm{C}\right.$ and $\left.1135^{\circ} \mathrm{C}\right)$ are shown in Figures 5.1A-C. It is observed that at lower temperature, oxygen is available for reaction almost throughout the catalyst length. On the other hand, at high temperature, oxygen is consumed close to the catalyst entrance, forming $\mathrm{H}_{2} \mathrm{O}$ and $\mathrm{CO}_{2}$. The $\mathrm{CO}_{2}$ profile shows a maximum, thus indicating subsequent consumption in other reactions. Computation of the overall rates for production/consumption of species and their relative rate ratios revealed four reaction zones over the catalyst length, which will be further elucidated in Sections 5.1-5.4. RPA schematics for those zones are shown in Figures 5.2-5.8. 

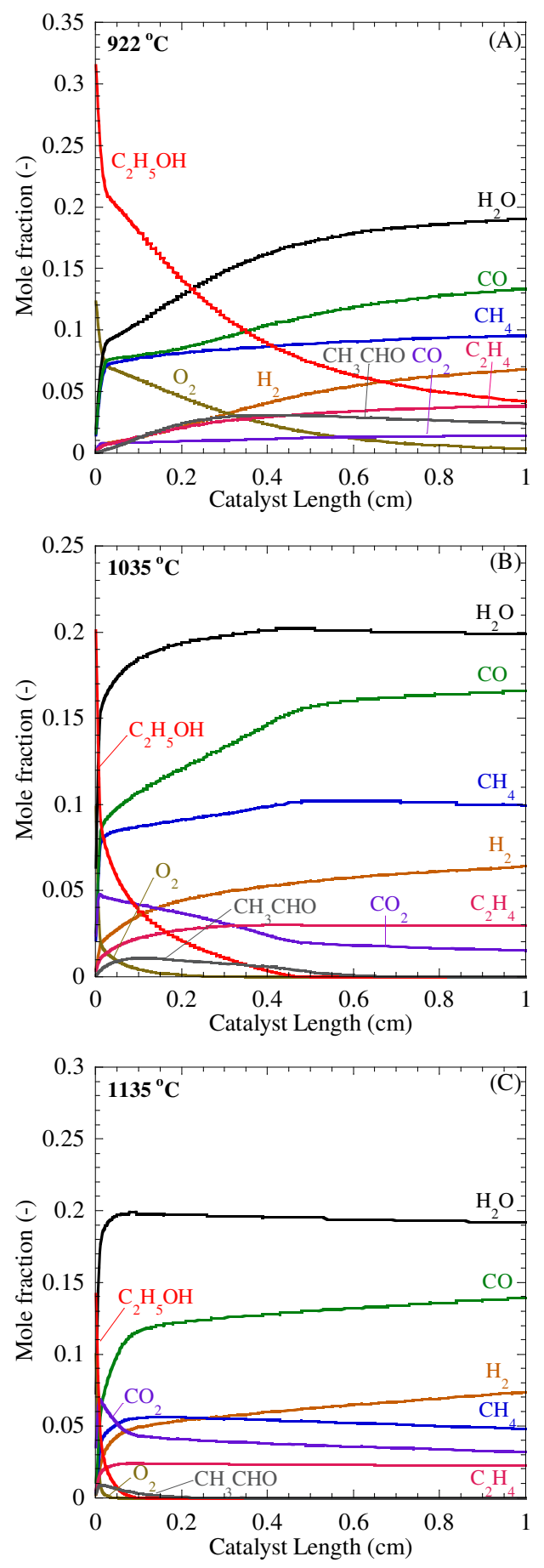

Figure 5.1 :Simulated mole fraction profiles for ethanol partial oxidation at $922{ }^{\circ} \mathrm{C}(\mathrm{A})$, $1035^{\circ} \mathrm{C}(\mathrm{B})$, and $1135^{\circ} \mathrm{C}(\mathrm{C})$. Operating conditions are the same as those in Figure 4.1. 


\subsection{Zone 1: Ethanol Dehydration and Oxidation}

The first zone at the beginning of the catalyst is dehydration and oxidation of ethanol, leading to high levels of $\mathrm{H}_{2} \mathrm{O}, \mathrm{CH}_{4}, \mathrm{CO}, \mathrm{CO}_{2}$, and $\mathrm{H}_{2}$, as well as some $\mathrm{C}_{2} \mathrm{H}_{4}$. A schematic of the dominant reactions using RPA is shown in Figure 5.2. Ethanol goes through two dehydration reactions creating water and either $\mathrm{C}_{2} \mathrm{H}_{4}{ }^{*}(\sim 6 \%)$, which desorbs into the gas phase, or $\mathrm{CHCH}_{3}{ }^{*}(\sim 94 \%)$ on the surface. $\mathrm{CHCH}_{3}{ }^{*}$ undergoes a carbon-carbon scission, to form $\mathrm{CH}^{*}$ and $\mathrm{CH}_{3}{ }^{*}$ species. Most $\mathrm{CH}_{3}{ }^{*}(\sim 80 \%)$ combines with hydrogen to form $\mathrm{CH}_{4}$; this is evident from the mole fraction profiles (Figures 6A-C). At the inlet, there is a large amount of oxygen available, which adsorbs dissociatively and participates in dehydrogenation of $\mathrm{CH}_{\mathrm{x}}{ }^{*}$ species. The remaining $\mathrm{CH}_{3}{ }^{*}$ can undergo oxidation with $\mathrm{O}^{*}$ and $\mathrm{OH}^{*}$ species, or thermal dehydrogenation to form $\mathrm{CH}_{2}{ }^{*} \cdot \mathrm{CH}_{2}{ }^{*}$ undergoes thermal dehydrogenation to form $\mathrm{CH}^{*}$, contributing to about $15 \%$ of $\mathrm{CH}^{*}$ on the surface; the rest originates from $\mathrm{CHCH}_{3}{ }^{*}$ scission. About half of $\mathrm{CH}^{*}$ is oxidized by $\mathrm{O}^{*}$ to form $\mathrm{CO}^{*}$ and the other half undergoes further thermal dehydrogenation to $C^{*}$. The $C^{*}$ species is oxidized via either $\mathrm{O}^{*}$ or $\mathrm{OH}^{*}$ to form $\mathrm{CO}^{*}$. Most $\mathrm{CO}^{*}(\sim 70 \%)$ desorbs into the gas phase and the remaining undergoes further oxidation to $\mathrm{CO}_{2}{ }^{*}$, all of which desorbs into the gas phase. The decrease in ethanol and spike in $\mathrm{CO}_{2}$ is clearly seen in the mole fraction profiles at high temperatures, while the spike is less pronounced at low temperatures. This chemistry is valid until oxygen is completely consumed. A small percentage of ethanol is consumed in the gas phase to produce $\mathrm{CH}_{3} \mathrm{CHO}$, which participates in the subsequent zones. The RPA of the gas is shown in Figure 5.3. Based on the rate ratios for the dominant species, the approximate stoichiometry of the overall reaction in this zone at $1035{ }^{\circ} \mathrm{C}$ is as follows (some reactions do not balance exactly due to rounding and the stoichiometry may be different at other temperatures): 
Surface chemistry $(90.3 \%)$ :

$$
\mathrm{C}_{2} \mathrm{H}_{5} \mathrm{OH}+0.8 \mathrm{O}_{2} \rightarrow 1.1 \mathrm{H}_{2} \mathrm{O}+0.8 \mathrm{CH}_{4}+0.8 \mathrm{CO}+0.3 \mathrm{CO}_{2}+0.2 \mathrm{H}_{2}+0.06 \mathrm{C}_{2} \mathrm{H}_{4}
$$

Gas chemistry $(9.7 \%)$ :

$$
\mathrm{C}_{2} \mathrm{H}_{5} \mathrm{OH}+0.3 \mathrm{O}_{2} \rightarrow 0.6 \mathrm{H}_{2} \mathrm{O}+0.4 \mathrm{CH}_{3} \mathrm{CHO}+0.4 \mathrm{C}_{2} \mathrm{H}_{4}+0.2 \mathrm{CH}_{2} \mathrm{O}+0.2 \mathrm{CH}_{4}+0.2 \mathrm{H}_{2}+
$$

$0.1 \mathrm{H}_{2} \mathrm{O}_{2}$

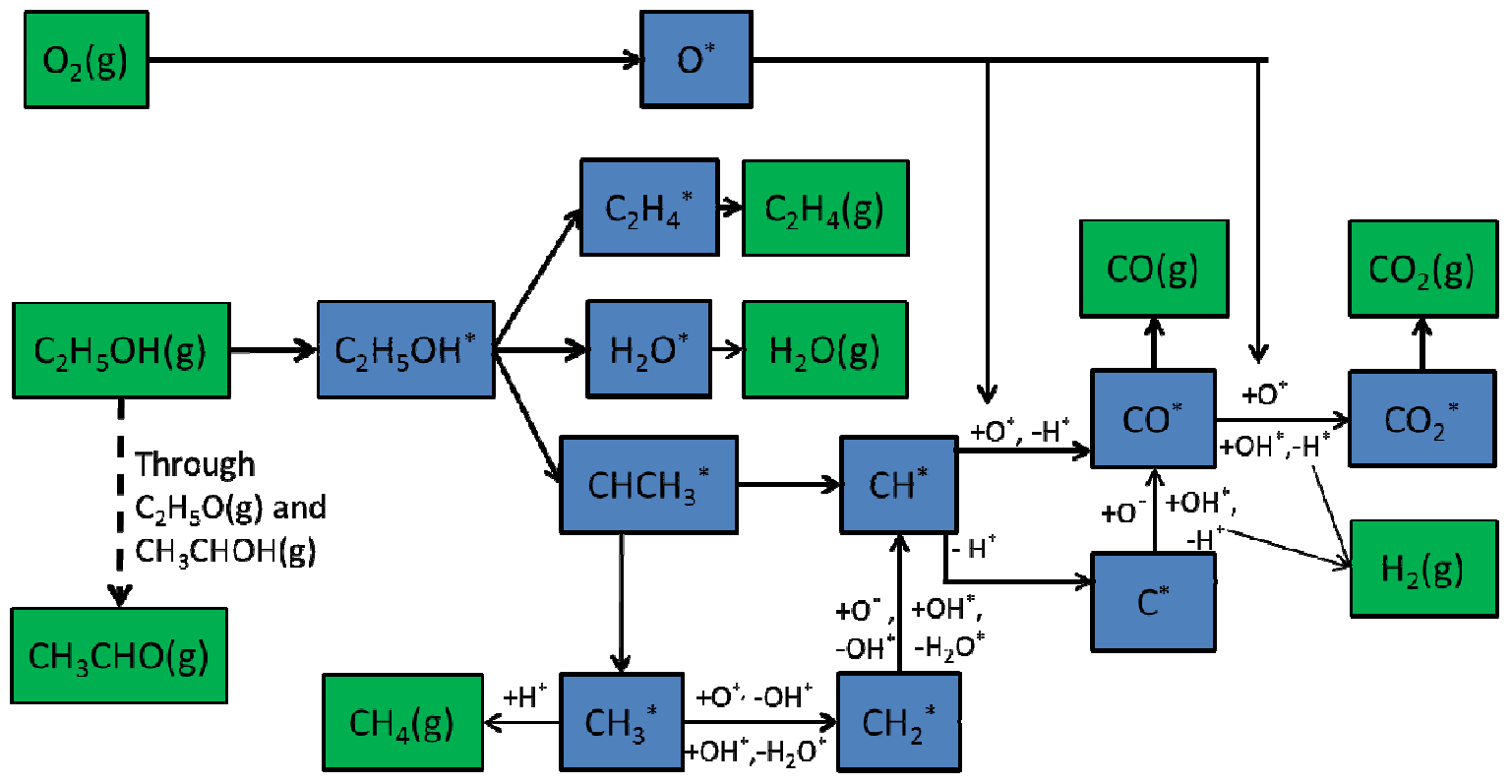

Figure 5.2: Schematic of the primary surface reaction pathways in the first zone (ethanol dehydration and oxidation). Green boxes designate gas phase reactants and final products, whereas blue boxes designate surface species. 


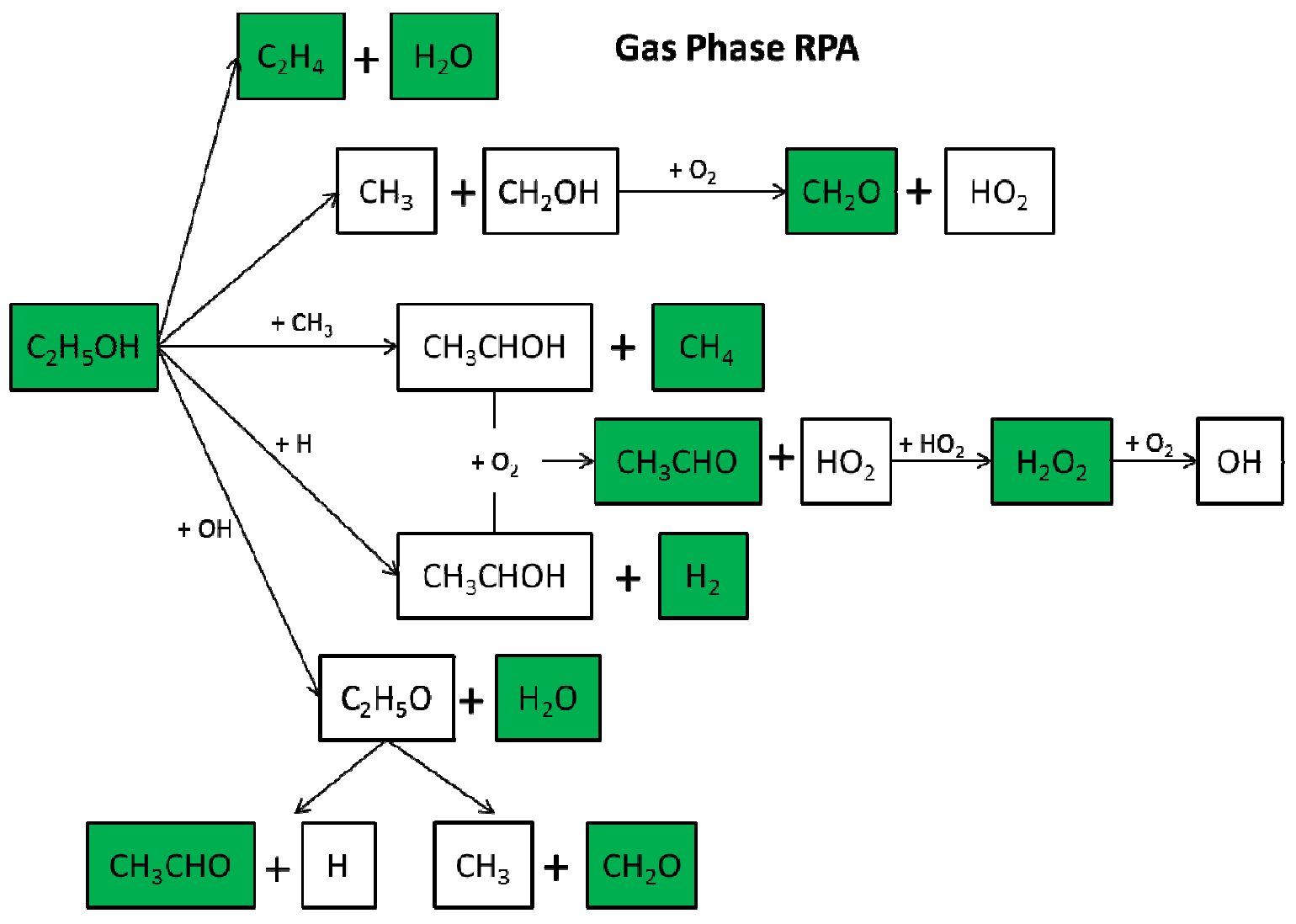

Figure 5.3: Schematic of the primary gas phase reaction pathways in the first zone (ethanol dehydration and oxidation). Green boxes designate reactants and final products, whereas white boxes designate intermediate species. 


\subsection{Zone 2: Transition: Ethanol Oxidation and Dry Reforming}

After the first zone of ethanol dehydration and oxidation, gas phase $\mathrm{CO}_{2}$ (formed from oxidation) adsorbs back on the surface (Figures 5.1A-C). A schematic of the dominant surface reactions using RPA is shown in Figure 5.4, however it should be noted that gas phase chemistry is important in this zone, and the gas phase RPA is shown in Figure 5.5. Surface chemistry in this transition zone is similar to the previous zone, including dehydration of ethanol, except $\mathrm{CO}_{2}$ provides re-adsorbed $\mathrm{CO}_{2}{ }^{*}$ for reaction with $\mathrm{C}^{*}$ (originating from $\left.\mathrm{CHCH}_{3}{ }^{*}\right)$ to form $\mathrm{CO}^{*}$ through the reverse Boudouard reaction $\left(\mathrm{C}^{*}+\right.$ $\mathrm{CO}_{2}{ }^{*} \rightarrow 2 \mathrm{CO}^{*}$ ), followed by $\mathrm{CO}^{*}$ desorption. The consumption of $\mathrm{CO}_{2}$ and spike in $\mathrm{CO}$ is seen in the mole fraction profiles as well. In this transition zone, the rate of $\mathrm{O}_{2}$ consumption decreases as it becomes depleted, whereas the rate of $\mathrm{CO}_{2}$ consumption increases. The boundaries of this zone are defined as when $\mathrm{CO}_{2}$ begins to adsorb on the surface until the rate of $\mathrm{O}_{2}$ consumption is less than $1 \%$ compared to that of $\mathrm{CO}_{2}$. Based on the rate ratios for the dominant species, the approximate stoichiometry of the reaction when $\mathrm{O}_{2}$ and $\mathrm{CO}_{2}$ have similar rates in this zone at $1035^{\circ} \mathrm{C}$ is as follows:

Surface chemistry $(26.9 \%)$ :

$\mathrm{C}_{2} \mathrm{H}_{5} \mathrm{OH}+0.5 \mathrm{CO}_{2}+0.3 \mathrm{O}_{2} \rightarrow 1.6 \mathrm{CO}+\mathrm{H}_{2} \mathrm{O}+0.8 \mathrm{CH}_{4}+0.3 \mathrm{H}_{2}+0.06 \mathrm{C}_{2} \mathrm{H}_{4}$

Gas chemistry $(73.1 \%)$ :

$\mathrm{C}_{2} \mathrm{H}_{5} \mathrm{OH}+0.3 \mathrm{O}_{2} \rightarrow 0.9 \mathrm{H}_{2} \mathrm{O}+0.6 \mathrm{H}_{2}+0.4 \mathrm{C}_{2} \mathrm{H}_{4}+0.4 \mathrm{CO}+0.1 \mathrm{CH}_{4}+0.1 \mathrm{CO}_{2}+0.1 \mathrm{CH}_{3} \mathrm{CHO}+$ $0.1 \mathrm{CH}_{2} \mathrm{CO}$ 


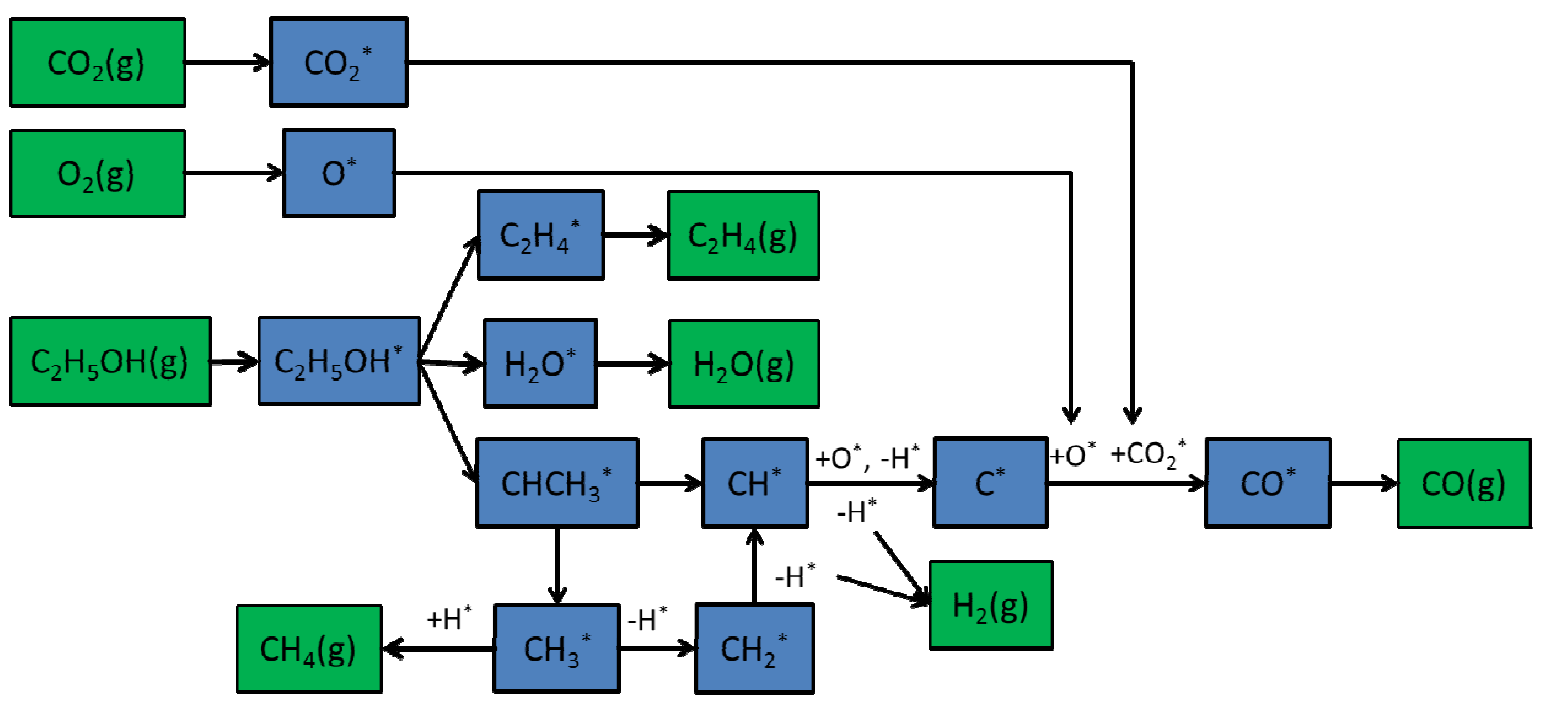

Figure 5.4: Schematic of the primary surface reaction pathways in the second zone (transition between ethanol oxidation and dry reforming). Green boxes designate gas phase reactants and final products, whereas blue boxes designate surface species. 


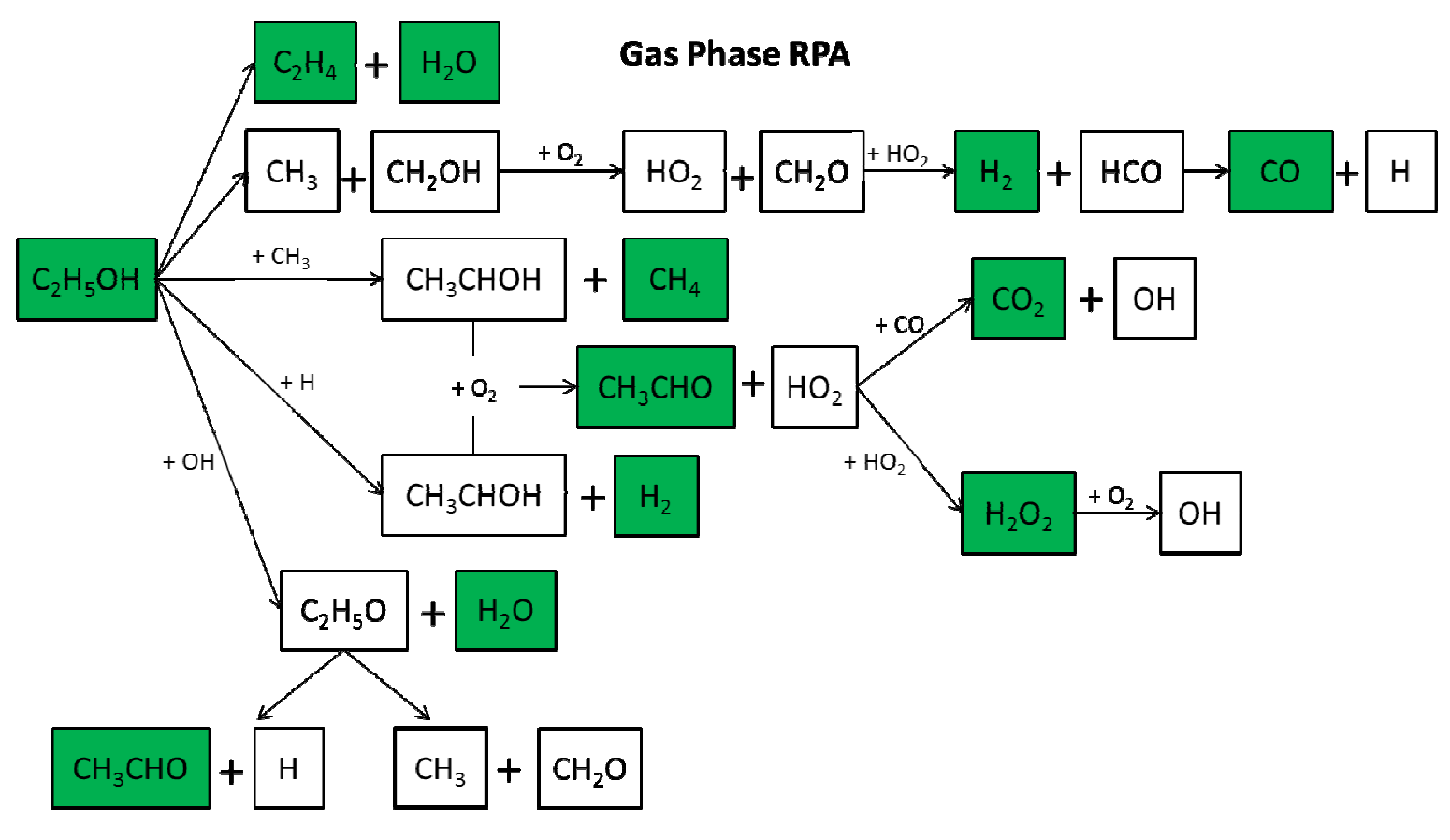

Figure 5.5: Schematic of the primary gas phase reaction pathways in the second zone (transition between ethanol oxidation and dry reforming). Green boxes designate reactants and final products, whereas white boxes designate intermediate species. 


\subsection{Zone 3: Dry Reforming of Ethanol}

In this zone, ethanol undergoes the same dehydration reactions as in the first oxidation zone, but after the $\mathrm{C}-\mathrm{C}$ scission of $\mathrm{CHCH}_{3}{ }^{*}$ on the surface, the $\mathrm{CH}^{*}$ and $\mathrm{CH}_{3}{ }^{*}$ species now undergo only thermal dehydrogenations, due to lack of $\mathrm{O}^{*}$. A schematic of the dominant reactions using RPA is shown in Figure 5.6. As described before, $\mathrm{C}^{*}$ reacts with the adsorbed $\mathrm{CO}_{2}{ }^{*}$, and forms $\mathrm{CO}^{*}$ through the reverse Boudouard reaction. In this region, most of the $\mathrm{CH}_{3}{ }^{*}$ formed on the surface from $\mathrm{CHCH}_{3}{ }^{*}$ reacts with hydrogen to form $\mathrm{CH}_{4}$, and the majority of the $\mathrm{CO}$ formed originates from $\mathrm{CH}^{*}$ (via $\mathrm{C}^{*}$ ). There is a small contribution of acetaldehyde formed from ethanol in the gas phase of the first and second zone. $\mathrm{CH}_{3} \mathrm{CHO}$ decomposes to $\mathrm{CH}_{3} \mathrm{CO}^{*}$, which subsequently decomposes to provide $\mathrm{CH}_{3}{ }^{*}$ and $\mathrm{CO}^{*}$. Though the gas phase contribution is small, a schematic of the gas phase RPA is shown in Figure 5.7. Based on the rate ratios for the dominant species, the approximate stoichiometry of the overall reaction in this zone at $1035^{\circ} \mathrm{C}$ is as follows:

Surface chemistry $(93.8 \%)$ :

$\mathrm{C}_{2} \mathrm{H}_{5} \mathrm{OH}+1.3 \mathrm{CO}_{2}+0.6 \mathrm{CH}_{3} \mathrm{CHO} \rightarrow 3.2 \mathrm{CO}+1.2 \mathrm{CH}_{4}+\mathrm{H}_{2} \mathrm{O}+0.7 \mathrm{H}_{2}+0.06 \mathrm{C}_{2} \mathrm{H}_{4}$

Gas chemistry (6.2\%):

$\mathrm{C}_{2} \mathrm{H}_{5} \mathrm{OH}+0.7 \mathrm{CH}_{3} \mathrm{CHO}+0.5 \mathrm{CH}_{2} \mathrm{O}+0.2 \mathrm{C}_{2} \mathrm{H}_{6} \rightarrow 1.6 \mathrm{CO}+1.6 \mathrm{H}_{2}+0.7 \mathrm{H}_{2} \mathrm{O}+0.7 \mathrm{C}_{2} \mathrm{H}_{4}+0.6$ $\mathrm{CH}_{4}$ 


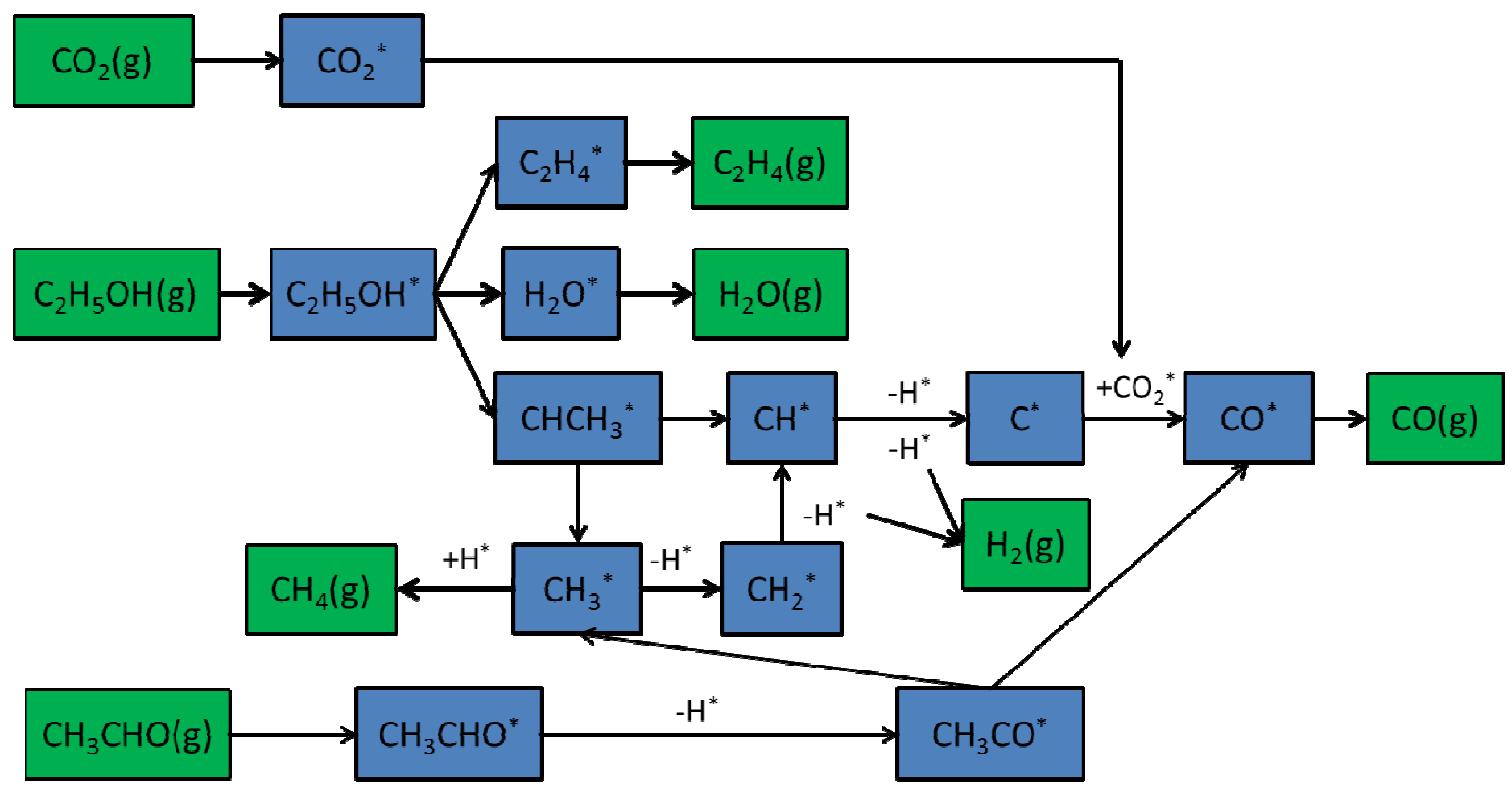

Figure 5.6: Schematic of the primary surface reaction pathways in the third zone (dry reforming of ethanol). Green boxes designate gas phase reactants and final products, whereas blue boxes designate surface species. 

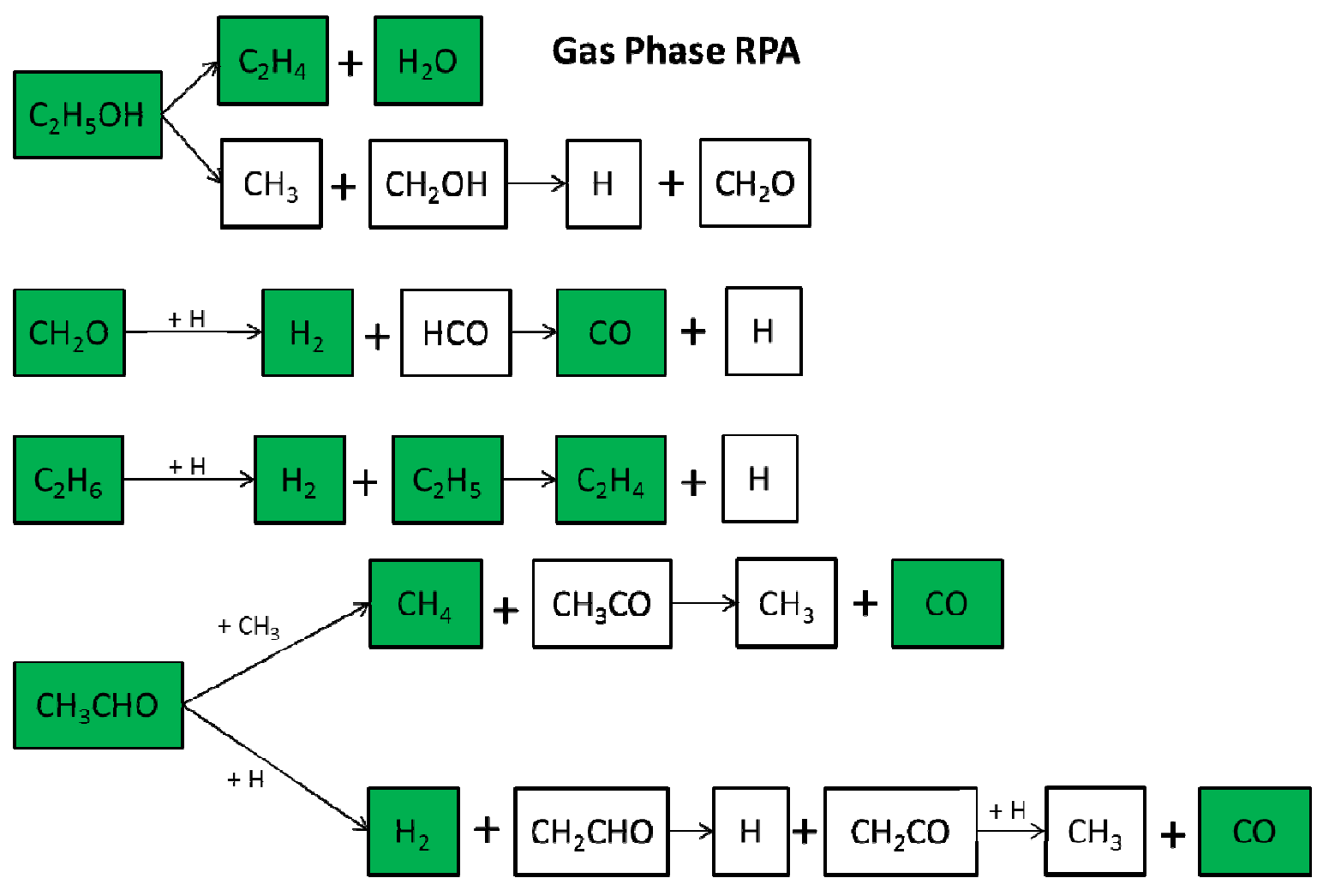

Figure 5.7: Schematic of the primary gas phase reaction pathways in the third zone (dry reforming of ethanol). Green boxes designate reactants and final products, whereas white boxes designate intermediate species.

For the lowest temperatures simulated, viz., $922{ }^{\circ} \mathrm{C}$ and $985{ }^{\circ} \mathrm{C}$, this zone and the following zone are not observed as oxygen and ethanol are never completely converted or consumed at these temperatures. This is observed from the mole fraction profiles as well (Figure 5.1A). 


\subsection{Zone 4: Dry and Steam Reforming of Methane}

At higher temperatures, ethanol begins to become depleted, and methane formed during the first three zones adsorbs back to the surface (Figures 5.1B-C). This transition is almost immediate and there is not a significantly long "transition zone" as in zone 2 . The beginning of the zone is defined as when ethanol consumption is less than $1 \%$ in comparison to that of methane. A schematic of the dominant reactions using RPA is shown in Figure 5.8. $\mathrm{CH}_{4}$ re-adsorption provides $\mathrm{CH}_{3}{ }^{*}$ species on the surface. The $\mathrm{CH}_{\mathrm{x}}{ }^{*}$ species undergo thermal dehydrogenations until $\mathrm{C}^{*}$ is formed, most of which then reacts with adsorbed $\mathrm{CO}_{2}{ }^{*}$ to form $\mathrm{CO}^{*}$, followed by desorption to $\mathrm{CO}$. In this zone, water formed in the first three zones also re-adsorbs on the surface and contributes (via $\mathrm{OH}^{*}$ ) to about $10 \%$ of $\mathrm{C}^{*}$ consumption. This is also evident from the slight decrease in the $\mathrm{H}_{2} \mathrm{O}$ mole fraction profiles at high temperatures (Figures 5.1B-C). $\mathrm{CH}_{3} \mathrm{CHO}$ formed in the first and second zone continues to adsorb on the surface and decompose to $\mathrm{CH}_{3} \mathrm{CO}^{*}$, which further decomposes to provide about $20 \%$ of $\mathrm{CH}_{3}{ }^{*}$ and $10 \%$ of $\mathrm{CO}^{*}$ on the surface. Based on the rate ratios for the dominant species, the approximate stoichiometry of the overall reaction when ethanol and methane has similar rates in this zone at $1035^{\circ} \mathrm{C}$ is as follows:

Surface chemistry (100\%):

$\mathrm{CH}_{4}+1.3 \mathrm{CO}_{2}+0.3 \mathrm{CH}_{3} \mathrm{CHO}+0.1 \mathrm{H}_{2} \mathrm{O} \rightarrow 2.9 \mathrm{CO}+2.9 \mathrm{H}_{2}$ 


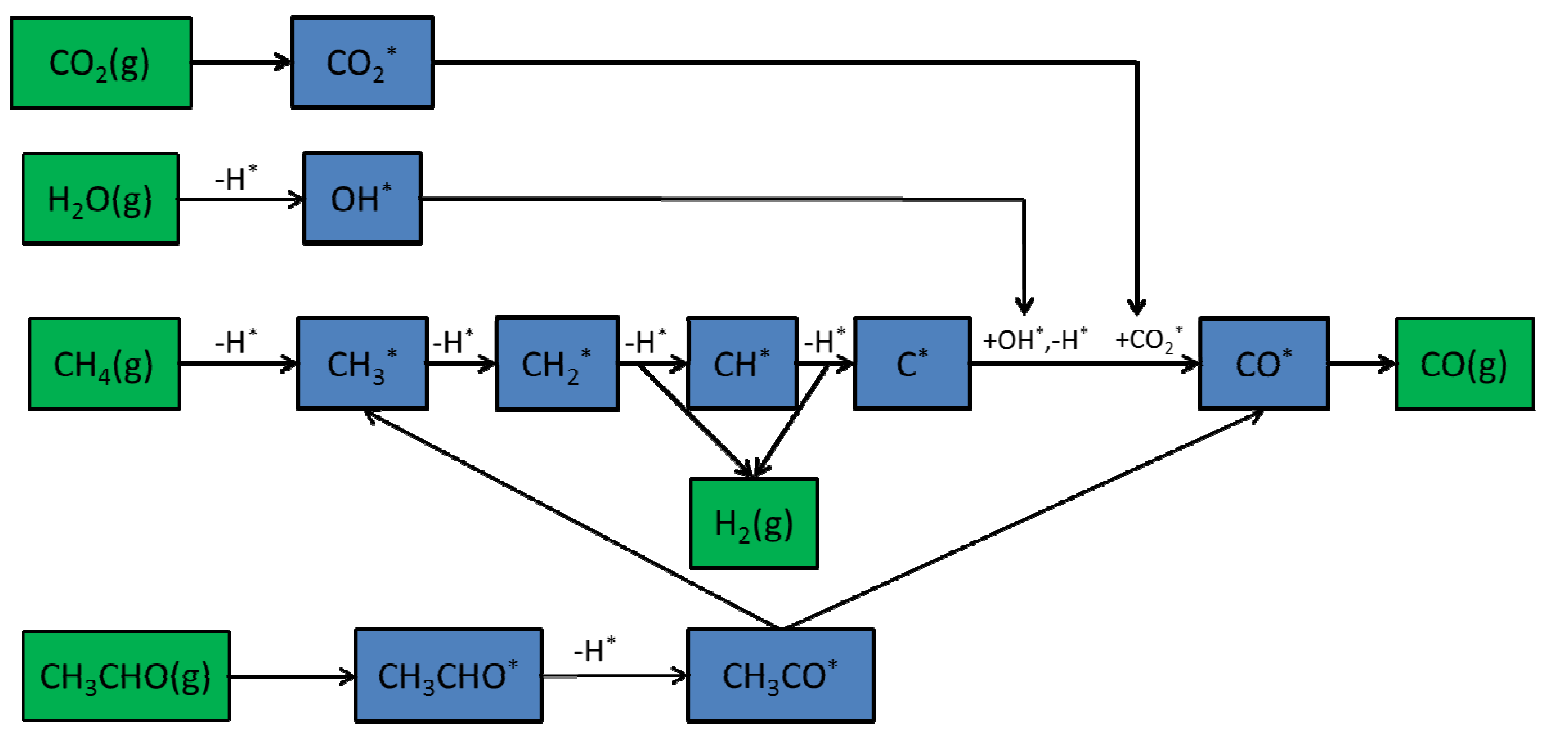

Figure 5.8: Schematic of the primary surface reaction pathways in the fourth zone (dry and steam reforming of methane). Green boxes designate gas phase reactants and final products, whereas blue boxes designate surface species.

For the lowest temperatures simulated, viz., $922{ }^{\circ} \mathrm{C}$ and $985^{\circ} \mathrm{C}$, where oxygen and ethanol are never depleted, $\mathrm{CH}_{4}$ does not re-adsorb from the gas phase back to the surface and its mole fraction continues to increase. This is observed from the mole fraction profiles as well (Figure 5.1A).

\subsection{Summary of Reaction Zones}

The reaction zones are well demonstrated by the axial profiles of surface species coverages as well, which are shown at $922{ }^{\circ} \mathrm{C}, 1035^{\circ} \mathrm{C}$ and $1135^{\circ} \mathrm{C}$ in Figure 5.9A-C. At lower temperatures, $\mathrm{CH}^{*}$ is the most abundant reaction intermediate (MARI) on the surface. At higher temperatures, $\mathrm{CH}^{*}$ initially covers the surface, then decreases as it is consumed by reaction with $\mathrm{O}^{*}$ in the initial oxidation zone. $\mathrm{CH}^{*}$ continues to have low coverage at higher temperatures where the forward reaction rate for $\mathrm{CH}^{*}$ dehydrogenation is higher than the backward reaction. 

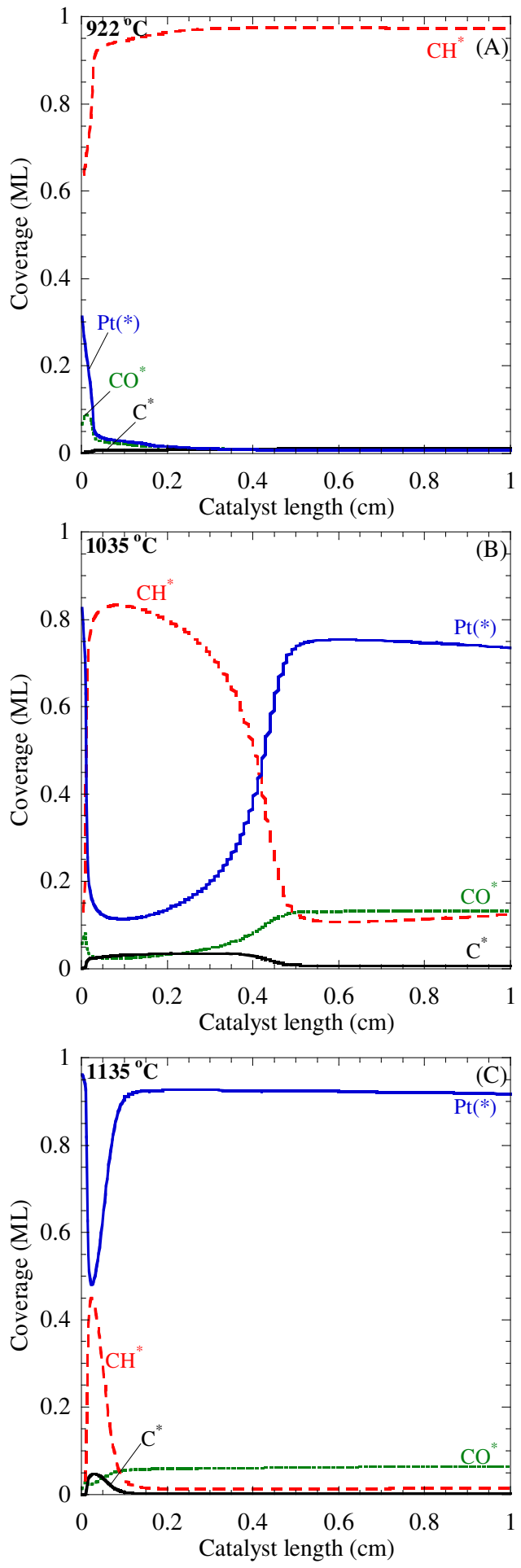

Figure 5.9: Simulated coverage profiles for ethanol partial oxidation at $922{ }^{\circ} \mathrm{C}, 1035$ ${ }^{\circ} \mathrm{C}$, and $1135{ }^{\circ} \mathrm{C}$. 
Figure 5.10 shows the locations of the reaction zones. Simulations at high temperatures displayed four reaction zones (including one transition zone from oxidation to dry reforming). However, we observed only two reaction zones at low temperatures $\left(922^{\circ} \mathrm{C}\right.$ and $\left.985^{\circ} \mathrm{C}\right)$ in which oxygen and ethanol are not completely consumed. Typically, two reaction zones have been observed in earlier literature studies for partial oxidation of $\mathrm{CH}_{4}$ - an oxidation zone followed by reforming [16,72]. For higher C-containing species such as ethanol, the presence of up to four zones indicates the complexity of the overall chemistry.

Despite the role of $\mathrm{CH}_{3} \mathrm{CHO}$ and $\mathrm{H}_{2} \mathrm{O}$ in zone 4, it should be noted their stoichiometric coefficients are low. Therefore, neglecting these smaller contributions, zone 4 could also be approximated as a zone of only dry reforming of $\mathrm{CH}_{4}$.

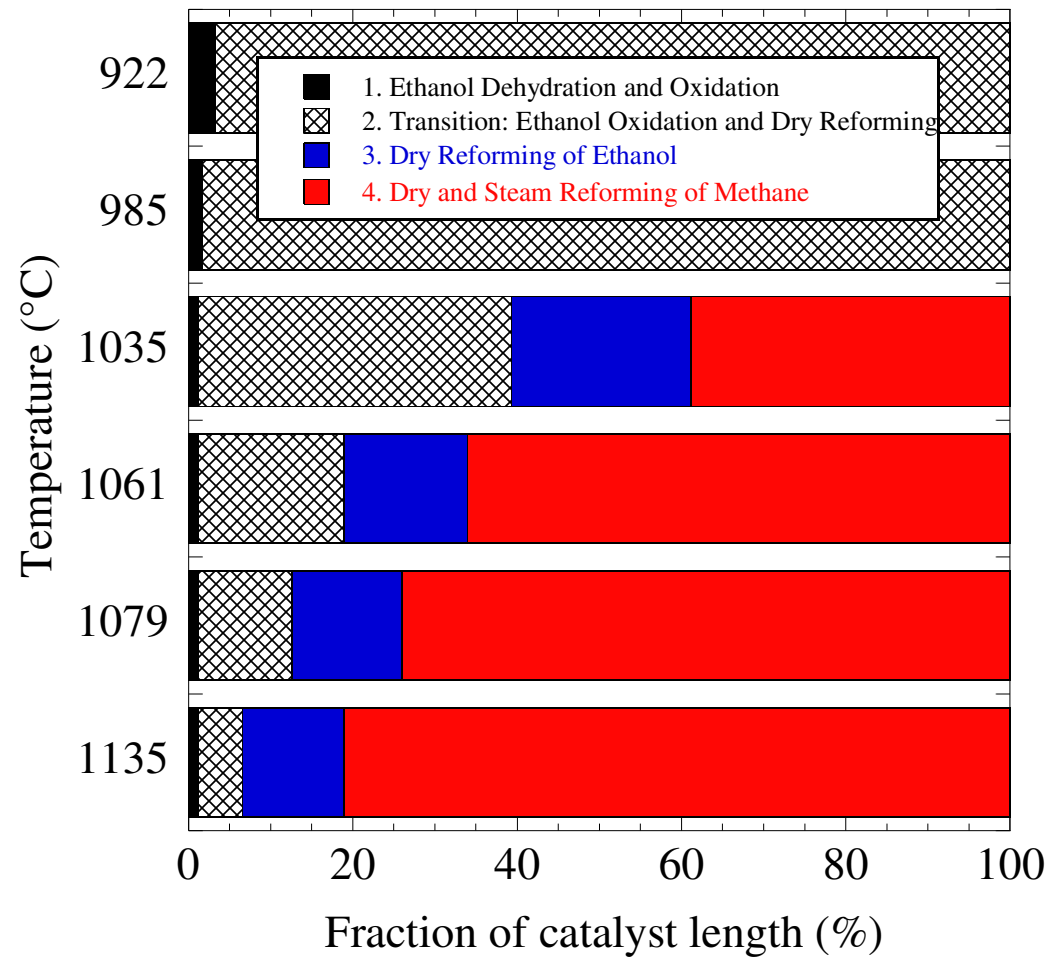

Figure 5.10: Distribution of the four reaction zones at various reaction temperatures 


\subsection{Reaction Order Analysis}

Pairwise sensitivity analysis showed that oxygen adsorption/desorption is the rate determining step (RDS) for ethanol conversion in the initial oxidation zone of the reactor. In the initial oxidation zone, the reaction orders with respect to oxygen and ethanol are 3.1 and -2.8 , respectively. Such analysis could be conducted for various responses (product selectivities) in all the reaction zones. However, due to the presence of multiple reaction zones and the changes in operating conditions, the RDS and reaction orders also vary. Rigorous validation of the predicted RDS and reaction orders is desirable, but it requires additional experimental data and analysis. 


\section{CHAPTER 6: Mechanism Validation against Dry Reforming Experiments}

The mechanistic analysis presented in the previous section reveals that dry reforming is a major component in three of the four reaction zones (Figure 5.10). Therefore, it is critical to ensure that the mechanism is rigorously validated against additional dry reforming experiments. The $\mathrm{CH}_{4}$ dry reforming conversion data reported in typical literature studies is close to being equilibrium limited (e.g., [88,89]). Therefore, we carried out $\mathrm{CH}_{4}$ dry reforming experiments on $\mathrm{Pt}$ in our fixed bed setup. In this section, we report the experimental details followed by the mechanism predictions without adjusting any kinetic parameters. Brief analysis of the dry reforming reaction pathways is also presented.

\subsection{BenchCAT Fixed Bed Reactor Experiments}

Methane dry reforming experiments were carried out in the temperature range of 600-1000 ${ }^{\circ} \mathrm{C}$ with an Altamira BenchCAT fixed bed reactor system, shown in Figure 6.1. $5 \% \mathrm{Pt} / \mathrm{Al}_{2} \mathrm{O}_{3}$ powder catalyst was synthesized using incipient wetness impregnation from Pt salt $\left(\mathrm{PtCl}_{4}\right.$, Sigma Aldrich). The reaction was carried out on $115 \mathrm{mg}$ of catalyst loaded in a $0.34 \mathrm{~cm}$ i.d. quartz tube reactor inside a temperature controlled furnace. $\mathrm{CH}_{4}(100 \%$, Airgas) was supplied with $10 \% \mathrm{CO}_{2}$ in Argon using mass flow controllers. In the first set of experiments, gases were supplied at a $\mathrm{CH}_{4}: \mathrm{CO}_{2}$ molar ratio of $1: 1$, whereas the molar ratio was changed to $2: 1$ in the second set of experiments to provide additional data for mechanism validation. Products $\left(\mathrm{CO}, \mathrm{CO}_{2}, \mathrm{H}_{2}\right)$ and unreacted reactant $\left(\mathrm{CH}_{4}\right)$ analysis was carried out with an Agilent 3000A microGC equipped with a thermal conductivity detector. A condenser was used to remove any water formed before the product analysis; however no water formation was observed. 


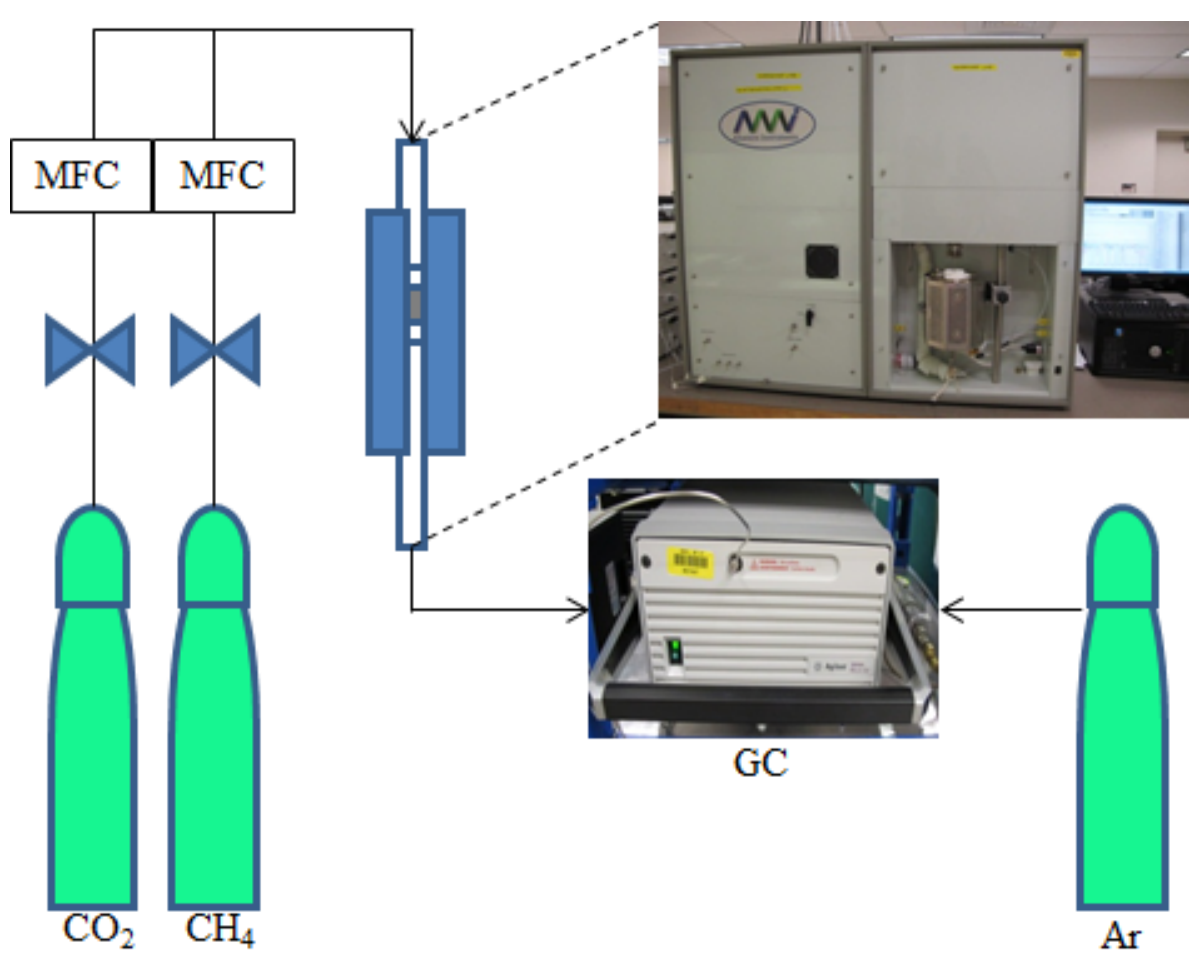

Figure 6.1: Schematic of the experimental setup used for methane dry reforming experiments.

\subsection{Mechanism Validation for Dry Reforming of Methane}

The surface reaction mechanism reported in Table 1 is also used to predict the $\mathrm{CH}_{4}$ dry reforming experimental data. Figure 6.2 shows the comparison of model predictions vs. experimental data of the conversions of $\mathrm{CH}_{4}$ and $\mathrm{CO}_{2}$ for the $1: 1$ molar ratio case. Equilibrium conversion (same for $\mathrm{CH}_{4}$ and $\mathrm{CO}_{2}$ in this case), calculated using Gaseq software [90], is also shown. 


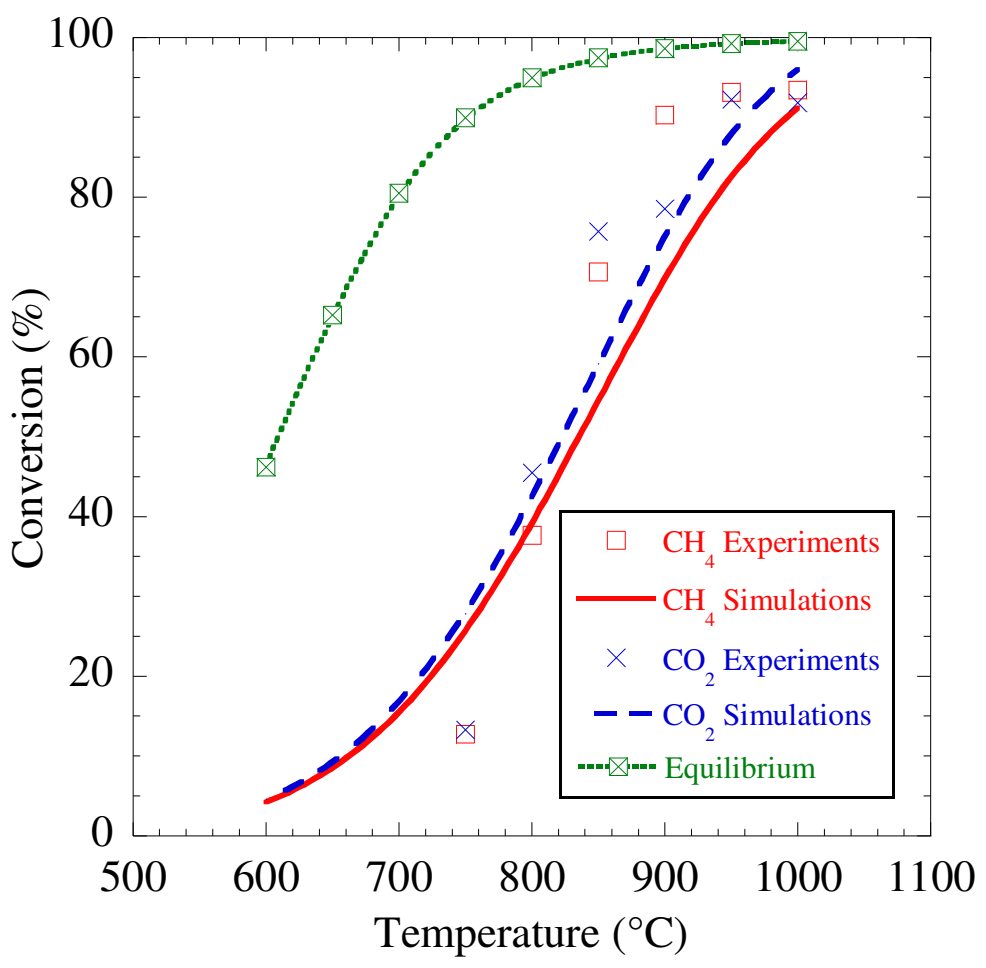

Figure 6.2: Mechanism validation against the methane dry reforming experimental data collected using the setup of Figure 6.1. The operating conditions are as follows: $55 \mathrm{sccm}$ of $9.1 \% \mathrm{CO}_{2}, 9.1 \% \mathrm{CH}_{4}$, and $81.8 \% \mathrm{AR}$, pressure $=1 \mathrm{~atm}$, weight of the $5 \% \mathrm{Pt} / \mathrm{Al}_{2} \mathrm{O}_{3}$ catalyst $=115 \mathrm{mg}$, velocity $=2.54 \mathrm{~cm} / \mathrm{sec}$, catalyst length $=7.8 \mathrm{~mm}$, and $\mathrm{A}_{\mathrm{c}} / \mathrm{V}_{\mathrm{R}}=600 \mathrm{~cm}^{-1}$. The 100-step optimized reaction mechanism is used in the simulations.

The experimental data is not equilibrium limited. Measured and predicted species mole fractions are shown in Figure 6.3A. For the 2:1 $\mathrm{CH}_{4}: \mathrm{CO}_{2}$ molar ratio, similar results are shown in Figure 6.3B. Overall, the microkinetic model predicts experimental data over 
the entire temperature range, for both the inlet composition conditions, without any further adjustment of kinetic parameters.

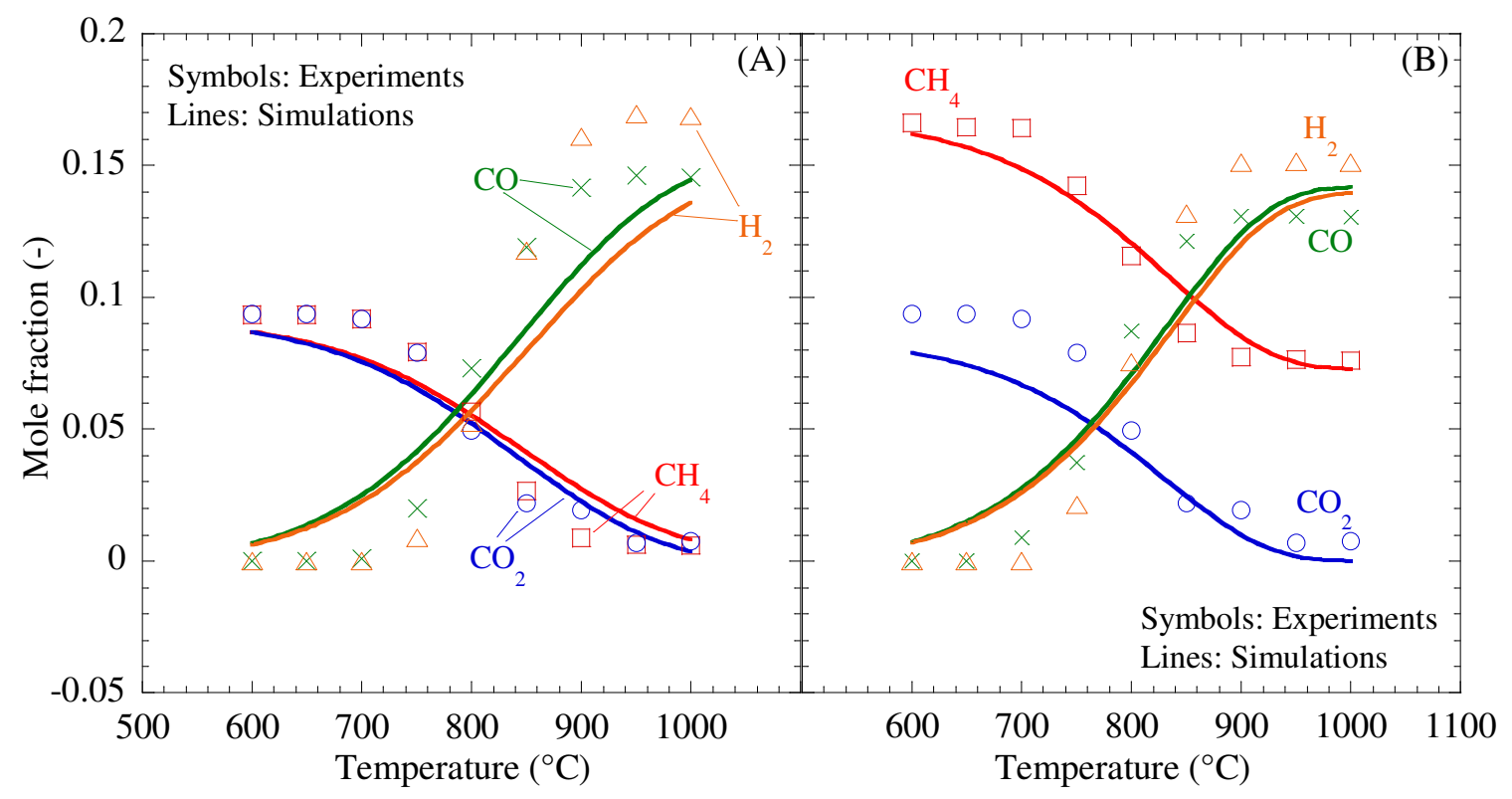

Figure 6.3: Comparison of model predicted and experimental mole fractions for methane dry reforming for inlet $\mathrm{CH}_{4}: \mathrm{CO}_{2}$ molar ratios of 1:1 (panel A) and 2:1 (panel B). Operating conditions for panel A are the same as those in Figure 6.2. Operating conditions for panel B are as follows: $60 \mathrm{sccm}$ of $8.3 \% \mathrm{CO}_{2}, 16.7 \% \mathrm{CH}_{4}$, and $75 \% \mathrm{AR}$, pressure $=1 \mathrm{~atm}$, weight of the $5 \%$ $\mathrm{Pt} / \mathrm{Al}_{2} \mathrm{O}_{3}$ catalyst $=115 \mathrm{mg}$, velocity $=2.75 \mathrm{~cm} / \mathrm{sec}$, catalyst length $=7.8$ $\mathrm{mm}$, and $\mathrm{A}_{\mathrm{c}} / \mathrm{V}_{\mathrm{R}}=600 \mathrm{~cm}^{-1}$. 


\subsection{Mechanistic Analysis and Dominant Reaction Pathways}

RPA of $\mathrm{CH}_{4}$ dry reforming is shown in Figure 6.4, where various pathways are emphasized depending on temperature. It is observed that the reaction proceeds via thermal dehydrogenations of $\mathrm{CH}_{\mathrm{x}}{ }^{*}$ species until $\mathrm{C}^{*}$ is formed. $\mathrm{C}^{*}$ then reacts with the adsorbed $\mathrm{CO}_{2}{ }^{*}$ through the reverse Boudouard reaction to form $\mathrm{CO}^{*}$, which desorbs. $\mathrm{H}^{*}$ atoms removed from the $\mathrm{CH}_{\mathrm{x}}{ }^{*}$ species combine to form gas phase $\mathrm{H}_{2}$. An alternative pathway for consumption of $\mathrm{CO}_{2}$ and production of $\mathrm{CO}$, is the reaction of $\mathrm{CO}_{2}{ }^{*}$ with $\mathrm{H}^{*}$ to form $\mathrm{CO}^{*}$ and $\mathrm{OH}^{*}$ (reverse Water-Gas Shift (RWGS)). This pathway accounts for $\sim 10 \%$ of $\mathrm{CO}_{2}$ consumption at $700{ }^{\circ} \mathrm{C}$ and decreases as the temperature increases until it does not occur at all at $1000{ }^{\circ} \mathrm{C}$. In this RPA, we find the dehydrogenation of $\mathrm{CH}_{3}{ }^{*}$ to $\mathrm{CH}_{2}{ }^{*}$ as the rate determining step, whereas all other reactions are in partial equilibrium. This aligns well with the reaction scheme proposed by Wei and Iglesia for dry reforming of methane on Pt [91], where they report that only the activation of the C-H bond is kinetically relevant and all other reactions are quasi-equilibrated. Our predicted reaction pathway corroborates this and specifically identifies the dehydrogenation of $\mathrm{CH}_{3}{ }^{*}$ as the dominant reaction for $\mathrm{C}-\mathrm{H}$ bond activation in methane dry reforming. 


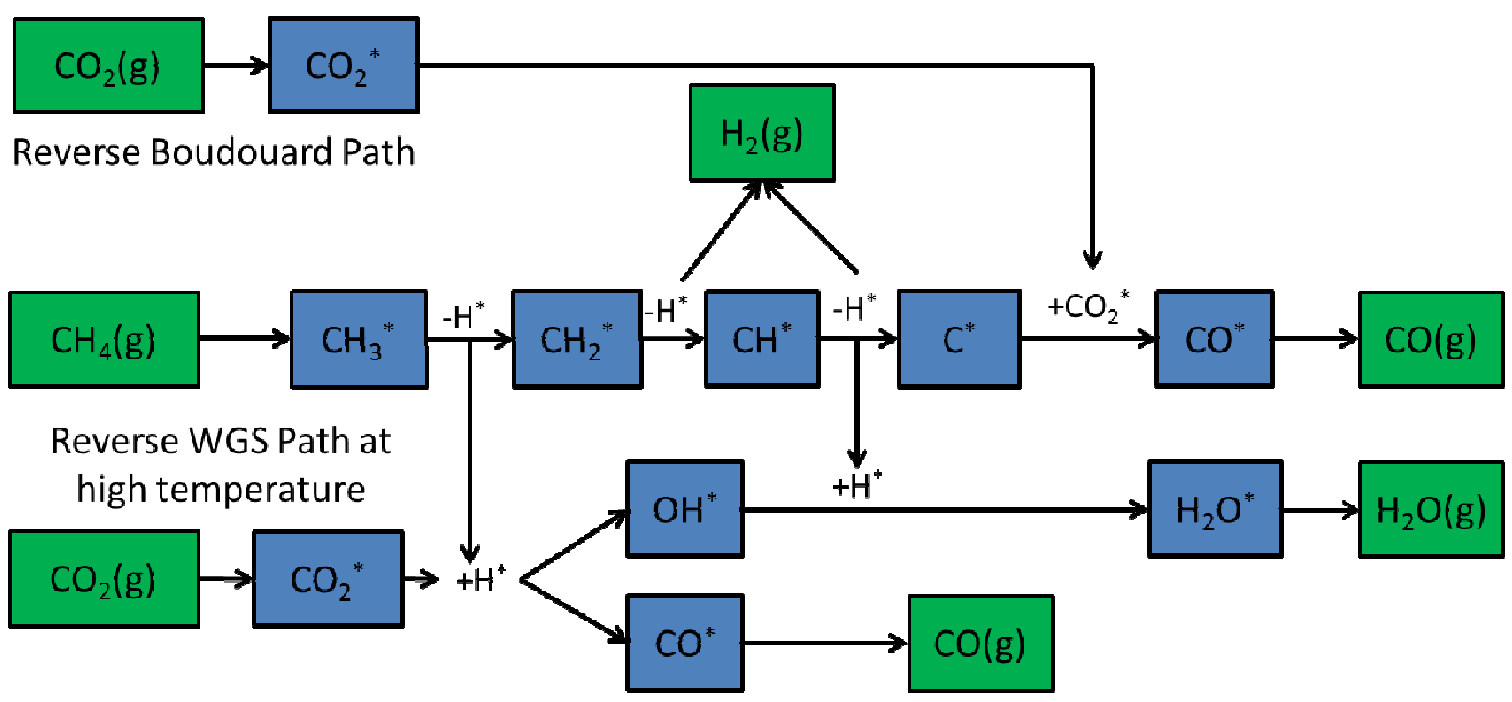

Figure 6.4: Schematic of the primary reaction pathways for the methane dry reforming.

Green boxes designate gas phase reactants and final products, whereas blue boxes designate surface species. 


\section{CONCLUSIONS}

In this work, we present the first microkinetic model for ethanol partial oxidation and reforming on $\mathrm{Pt}$, which is developed and validated against data from literature [22] and our experiments. Using a microkinetic modeling approach, a 100-step (50 reversible) thermodynamically consistent surface reaction mechanism is proposed. Starting from the initial kinetic parameter estimates, sensitivity analysis is carried out to identify the most important parameters that control the ethanol conversion and products selectivities. A total of seven kinetic parameters (pre-exponential factors) are adjusted during mechanism development. The model predicts ethanol conversion as well as multiple products $\left(\mathrm{C}_{2} \mathrm{H}_{4}\right.$, $\mathrm{CO}, \mathrm{H}_{2} \mathrm{O}$, and $\mathrm{CH}_{4}$ ) selectivities fairly well in most cases. A detailed mechanistic analysis of the reaction pathways reveals that there are four distinct reaction zones (ethanol dehydration and oxidation, transition from ethanol oxidation to dry reforming, dry reforming of ethanol, and dry and steam reforming of methane) over the catalyst length. As dry reforming dominates most of the catalytic chemistry, the surface reaction mechanism is further validated against our experimental data for dry reforming of $\mathrm{CH}_{4}$ on $\mathrm{Pt}$. Without any further adjustment of kinetic parameters, the microkinetic model predicts the dry reforming experimental data well. Despite the assumptions and limitations of the overall microkinetic modeling approach, this mechanism provides a starting point to gain a fundamental understanding of oxygenates reforming kinetics, with which detailed reactor and rational catalyst design could be carried out. 


\section{FUTURE WORK}

In general, the model would greatly benefit from further optimization and validation for a larger range of operating conditions than those discussed in this work. There also several ways in which the model can be improved to address the assumptions and limitations discussed earlier in this work. Minimizing the uncertainty arising from the assumptions used in this work would lead to a more rigorous model in which more confidence could be held in the output. This is important in applying the model to reactor design.

Removing model uncertainty would be greatly aided by more detailed information from both an experimental and computational aspect. Experimentally, a temperature profile would certainly be an asset to the model and remove uncertainty related to isothermal assumptions. Accounting of minor products, like acetaldehyde, would allow for further mechanism refinement and validation. Computationally, determination of binding energies via DFT would beneficial. Many binding energies are already taken from DFT, but from many different sources. To generate binding energies from the same method would be more astute for the model. DFT could also be utilized to generate more appropriate bond indices, or even fully determine activation energies, for important reactions.

The model can be further developed to consider more important catalyst aspects that are addressed with various assumptions in the current model. Currently, the model does not account for metal loading, which surely has a large effect on reaction performance. This might be addressed with a linear scaling of active catalyst area with metal loading. The mechanism does not consider the metal support, which might be 
incorporated by added reactions with support as well as considering support in the determination of reactant binding energies. The mechanism also does not account for deactivation or coking, which, though not an issue for $\mathrm{Pt}$, can be very problematic on other catalysts like Ni. Accounting for loss of active sites as they deactivate and adding coking reactions to the mechanism could make the model more realistic.

A mean-field approximation is used in this model but a kinetic Monte Carlo approach to modeling the metal surface as individual sites and is updated in real time would make the model more realistic [92]. This approach would allow for the proximity of adsorbates to be considered as well as different facets of the metal and their corresponding differences in binding energies.

The model could also be extended and validated for other catalysts. As the mechanism is dependent on binding energies of the metal catalyst, a well validated, robust mechanism could quickly assess materials for reforming performance. This would achieve the objective of computational studies, saving the time and cost of experimental testing. 


\section{REFERENCES}

[1] Deluga GA, Salge JR, Schmidt LD, Verykios XE. Renewable hydrogen from ethanol by autothermal reforming. Sci. 2004;303:993.

[2] Navarro RM, Pena MA, Fierro JLG. Hydrogen production reactions from carbon feedstocks: Fossil fuels and biomass. Chem. Rev. 2007;107:3952-91.

[3] Chiodo V, Freni S, Galvagno A, Mondello N, Frusteri F. Catalytic features of Rh and Ni supported catalysts in the steam reforming of glycerol to produce hydrogen. Applied Catalysis A: General 2010 6/15;381(1-2):1-7.

[4] Adhikari S, Fernando SD, Haryanto A. Hydrogen production from glycerol: An update. Energy Conversion and Management 2009 10;50(10):2600-4.

[5] Vaidya PD, Rodrigues AE. Glycerol reforming for Hydrogen production: A review. Chem. Eng. Technol. 2009;32(10):1463-9.

[6] Xuan J, Leung MKH, Leung DYC, Ni M. A review of biomass-derived fuel processors for fuel cell systems. Renewable and Sustainable Energy Reviews 2009 0;13(6-7):130113.

[7] Zhou C, Beltramini JN, Fan Y, Lu GQ. Chemoselective catalytic conversion of glycerol as a biorenewable source to valuable commodity chemicals. Chem. Soc. Rev. 2008;37(3):527-49.

[8] Demirbas A. Progress and recent trends in biofuels. Progress in Energy and Combustion Science 2007 2;33(1):1-18.

[9] Ni M, Leung DYC, Leung MKH. A review on reforming bio-ethanol for hydrogen production. Int J Hydrogen Energy 2007 10;32(15):3238-47.

[10] Turner J, Sverdrup G, Mann MK, Maness P, Kroposki B, Ghirardi M, et al. Renewable hydrogen production. Int. J. Energy Res. 2007;32(5):379-407.

[11] Vaidya PD, Rodrigues AE. Insight into steam reforming of ethanol to produce hydrogen for fuel cells. Chem.Eng.J. 2006 3/15;117(1):39-49.

[12] Haryanto A, Fernando S, Murali N, Adhikari S. Current status of hydrogen production techniques by steam reforming of ethanol: A review. Energy Fuels 2005;19(5):2098-106.

[13] Appari S, Janardhanan VM, Jayanti S, Maier L, Tischer S, Deutschmann O. Microkinetic modeling of $\mathrm{NH}_{3}$ decomposition on $\mathrm{Ni}$ and its application to solid oxide fuel cells. Chemical Engineering Science 2011 11/1;66(21):5184-91. 
[14] Chen D, Lodeng S, H., Holmen A. Hierarchial multiscale modeling of methane steam reforming reactions. Ind. Eng. Chem. Res. 2011;50:2600.

[15] Vlachos DG, Mhadeshwar AB, Kaisare NS. Hierarchical multiscale model-based design of experiments, catalysts, and reactors for fuel processing. Comput.Chem.Eng. 2006 9/12;30(10-12):1712-24.

[16] Mhadeshwar AB, Vlachos DG. Hierarchial multiscale mechanism development for methane partial oxidation and reforming and for thermal decomposition of oxygenates on Rh. J. Phys. Chem. B 2005;109(35):16819-35.

[17] Deshmukh SR, Mhadeshwar AB, Lebedeva MI, Vlachos DG. From Density Functional Theory to microchemical device homogenization: Model prediction of hydrogen production for portable fuel cells. International Journal for Multiscale Computational Engineering 2004;2(2):221-38.

[18] Schmal M, Cesar DV, Souza, Mariana M. V. M., Guarido CE. Drifts and TPD analyses of ethanol on Pt catalysts over $\mathrm{Al}_{2} \mathrm{O}_{3}$ and $\mathrm{ZrO}_{2}$-Partial oxidation of ethanol. Can. J. Chem. Eng. 2011;89(5):1-10-175.

[19] de Lima SM, da Silva AM, Jacobs G, Davis BH, Mattos LV, Noronha FB. New approaches to improving catalyst stability over Pt/ceria during ethanol steam reforming: $\mathrm{Sn}$ addition and $\mathrm{CO}_{2}$ co-feeding. Applied Catalysis B: Environmental 2010 6/7;96(3-4):38798.

[20] de Lima SM, Silva AM, da Cruz IO, Jacobs G, Davis BH, Mattos LV, et al. $\mathrm{H}_{2}$ production through steam reforming of ethanol over $\mathrm{Pt} / \mathrm{ZrO}_{2}, \mathrm{Pt} / \mathrm{CeO}_{2}$ and $\mathrm{Pt} / \mathrm{CeZrO}_{2}$ catalysts. Catalysis Today 2008 11;138(3-4):162-8.

[21] Dömök M, Tóth M, Raskó J, Erdöhelyi A. Adsorption and reactions of ethanol and ethanol-water mixture on alumina-supported Pt catalysts. Applied Catalysis B: Environmental 2007 1/15;69(3-4):262-72.

[22] Salge JR, Deluga GA, Schmidt LD. Catalytic partial oxidation of ethanol over noble metal catalysts. Journal of Catalysis 2005 10/1;235(1):69-78.

[23] Gutierrez A, Karinen R, Airaksinen S, Kaila R, Krause AOI. Autothermal reforming of ethanol on noble metal catalysts. Int J Hydrogen Energy 2011 7;36(15):8967-77.

[24] da Silva AM, de Souza KR, Jacobs G, Graham UM, Davis BH, Mattos LV, et al. Steam and $\mathrm{CO}_{2}$ reforming of ethanol over $\mathrm{Rh} / \mathrm{CeO}_{2}$ catalyst. Applied Catalysis B: Environmental 2011 2/1;102(1-2):94-109.

[25] Ciambelli P, Palma V, Ruggiero A. Low temperature catalytic steam reforming of ethanol. 1. The effect of the support on the activity and stability of Pt catalysts. Applied Catalysis B: Environmental 2010 4/26;96(1-2):18-27. 
[26] Ciambelli P, Palma V, Ruggiero A. Low temperature catalytic steam reforming of ethanol. 2. Preliminary kinetic investigation of $\mathrm{Pt} / \mathrm{CeO}_{2}$ catalysts. Applied Catalysis B: Environmental 2010 4/26;96(1-2):190-7.

[27] Hebben N, Diehm C, Deutschmann O. Catalytic partial oxidation of ethanol on alumina-supported rhodium catalysts: An experimental study. Applied Catalysis A: General 2010 11/20;388(1-2):225-31.

[28] Liguras DK, Kondarides DI, Verykios XE. Production of hydrogen for fuel cells by steam reforming of ethanol over supported noble metal catalysts. Applied Catalysis B: Environmental 2003 7/25;43(4):345-54.

[29] Song H, Mirkelamoglu B, Ozkan US. Effect of cobalt precursor on the performance of ceria-supported cobalt catalysts for ethanol steam reforming. Applied Catalysis A: General 2010 6/30;382(1):58-64.

[30] Song H, Ozkan US. The role of impregnation medium on the activity of ceriasupported cobalt catalysts for ethanol steam reforming. Journal of Molecular Catalysis A: Chemical 2010 3/1;318(1-2):21-9.

[31] Song H, Zhang L, Watson RB, Braden D, Ozkan US. Investigation of bio-ethanol steam reforming over cobalt-based catalysts. Catalysis Today 2007 12/15;129(3-4):34654.

[32] Fatsikostas AN, Verykios XE. Reaction network of steam reforming of ethanol over Ni-based catalysts. Journal of Catalysis 2004 7/25;225(2):439-52.

[33] Wanat EC, Suman B, Schmidt LD. Partial oxidation of alcohols to produce hydrogen and chemicals in millisecond-contact time reactors. Journal of Catalysis 2005 $10 / 1 ; 235(1): 18-27$.

[34] Skoplyak O, Menning CA, Barteau MA, Chen JG. Reforming of oxygenates for $\mathrm{H}_{2}$ production on $3 d / \operatorname{Pt}(111)$ bimetallic surfaces. Top. Catal. 2008;51(1-4):49-59.

[35] Vesselli E, Comelli G, Rosei R, Freni S, Frusteri F, Cavallaro S. Ethanol auto-thermal reforming on rhodium catalysts and initial steps simulation on single crystals under UHV conditions. Applied Catalysis A: General 2005 3/18;281(1-2):139-47.

[36] Benito M, Padilla R, Serrano-Lotina A, Rodríguez L, Brey JJ, Daza L. The role of surface reactions on the active and selective catalyst design for bioethanol steam reforming. J.Power Sources 2009 7/1;192(1):158-64.

[37] de Lima SM, da Cruz IO, Jacobs G, Davis BH, Mattos LV, Noronha FB. Steam reforming, partial oxidation, and oxidative steam reforming of ethanol over $\mathrm{Pt} / \mathrm{CeZrO}_{2}$ catalyst. Journal of Catalysis 2008 7/25;257(2):356-68. 
[38] Mattos LV, Noronha FB. Hydrogen production for fuel cell applications by ethanol partial oxidation on $\mathrm{Pt} / \mathrm{CeO}_{2}$ catalysts: The effect of the reaction conditions and reaction mechanism. Journal of Catalysis 2005 7/25;233(2):453-63.

[39] Jacobs G, Keogh RA, Davis BH. Steam reforming of ethanol over Pt/ceria with co-fed hydrogen. Journal of Catalysis 2007 1/25;245(2):326-37.

[40] Erdőhelyi A, Raskó J, Kecskés T, Tóth M, Dömök M, Baán K. Hydrogen formation in ethanol reforming on supported noble metal catalysts. Catalysis Today 2006 8/15;116(3):367-76.

[41] Rossi CCRS, Alonso CG, Antunes OAC, Guirardello R, Cardozo-Filho L. Thermodynamic analysis of steam reforming of ethanol and glycerine for hydrogen production. Int J Hydrogen Energy 2009 1;34(1):323-32.

[42] Wang W, Wang YQ. Thermodynamic analysis of steam reforming of ethanol for hydrogen generation. Int. J. Energy Res. 2008;32(15):1432-43.

[43] Fishtik I, Alexander A, Datta R, Geana D. A thermodynamic analysis of hydrogen production by steam reforming of ethanol via response reactions. Int J Hydrogen Energy 2000 1;25(1):31-45.

[44] Mas V, Bergamini ML, Baronetti G, Amadeo N, Laborde M. A kinetic study of ethanol steam reforming using a Nickel based catalyst. Top. Catal. 2008;51(1-4):39-48.

[45] Akpan E, Akande A, Aboudheir A, Ibrahim H, Idem R. Experimental, kinetic and 2-D reactor modeling for simulation of the production of hydrogen by the catalytic reforming of concentrated crude ethanol (CRCCE) over a Ni-based commercial catalyst in a packedbed tubular reactor. Chemical Engineering Science 2007 6;62(12):3112-26.

[46] Mathure PV, Ganguly S, Patwardhan AV, Saha RK. Steam reforming of ethanol using a commercial Nickel-based catalyst. Ind. Eng. Chem. Res. 2007;46(25):8471-9.

[47] Sahoo DR, Vajpai S, Patel S, Pant KK. Kinetic modeling of steam reforming of ethanol for the production of hydrogen over $\mathrm{Co} / \mathrm{Al}_{2} \mathrm{O}_{3}$ catalyst. Chem Eng J 2007;125:13947.

[48] Ferrin P, Simonetti D, Kandoi S, Kunkes E, Dumesic JA, Nørskov JK, et al. Modeling ethanol decomposition on transition metals: A combined application of scaling and Brønsted-Evans-Polanyi relations. J. Am. Chem. Soc. 2009;131:5809-15.

[49] Salciccioli M, Chen Y, Vlachos DG. Density Functional Theory-derived group additivity and linear scaling methods for prediction of oxygenate stability on metal catalysts: Adsorption of open-ring alcohol and polyol dehydrogenation intermediates on Pt-based metals. J. Phys. Chem. C 2010;114(47):20155-66. 
[50] Wang J, Lee CS, Lin MC. Mechanism of ethanol reforming: Theoretical foundations. J. Phys. Chem. C 2009;113(16):6681-8.

[51] Skoplyak O, Barteau MA, Chen JG. Ethanol and ethylene glycol on Ni/Pt(1 11 1) bimetallic surfaces: A DFT and HREELS study. Surf.Sci. 2008 12/1;602(23):3578-87.

[52] Pallassana V, Neurock M. Reaction paths in the hydrogenolysis of acetic acid to ethanol over Pd(111), Re(0001), and PdRe alloys. Journal of Catalysis 2002 7/25;209(2):289-305.

[53] Alcalá R, Mavrikakis M, Dumesic JA. DFT studies for cleavage of C-C and C-O bonds in surface species derived from ethanol on $\mathrm{Pt}(111)$. Journal of Catalysis 2003;218(1):178-90.

[54] Marinov NM. A detailed chemical kinetic model for high temperature ethanol oxidation. Int. J. Chem. Kinet. 1999;31(3):183-220.

[55] Christensen DO, Silveston PL, Croiset E, Hudgins RR. Production of hydrogen from the noncatalytic partial oxidation of ethanol. IndEngChemRes 2004;43(11):2636-42.

[56] Dumesic JA, Rudd DF, Aparicio LM, Rekoske JE, Treviño AA. The Microkinetics of Heterogeneous Catalysis USA: Oxford University Press; 1993.

[57] Maier L, Schadel B, Delgado KH, Tischer S, Deutschmann O. Steam reforming of methane over Nickel: Development of a multi-step surface reaction mechanism. Top. Catal. 2011;54(13-15):845-58.

[58] Maestri M, Vlachos DG, Beretta A, Groppi G, Tronconi E. Steam and dry reforming of methane on Rh: Microkinetic analysis and hierarchy of kinetic models. Journal of Catalysis 2008 10/25;259(2):211-22.

[59] Mhadeshwar AB, Winkler BH, Eiteneer B, Hancu D. Microkinetic modeling for hydrocarbon (HC)-based selective catalytic reduction (SCR) of $\mathrm{NO}_{\mathrm{x}}$ on a silver-based catalyst. Applied Catalysis B: Environmental 2009 7/3;89(1-2):229-38.

[60] Sjövall H, Blint RJ, Olsson L. Detailed kinetic modeling of $\mathrm{NH}_{3} \mathrm{SCR}$ over Cu-ZSM5. Applied Catalysis B: Environmental 2009 10/19;92(1-2):138-53.

[61] Hauptmann W, Votsmeier M, Gieshoff J, Vlachos DG, Drochner A, Vogel H. A fast approach to predictive models: NO-oxidation in exhaust gas aftertreatment systems. Top. Catal. 2009;52(13-20):1925-8.

[62] Prasad V, Karim AM, Ulissi Z, Zagrobelny M, Vlachos DG. High throughput multiscale modeling for design of experiments, catalysts, and reactors: Application to hydrogen production from ammonia. Chemical Engineering Science 2010 1/1;65(1):240-6. 
[63] Salciccioli M, Y. Chen Y, Vlachos DG. Microkinetic modeling and reduced rate expressions of ethylene hydrogenation and ethane hydrogenolysis on Platinum. Ind. Eng. Chem. Res. 2011;50(1):28-40.

[64] Madon RJ, Braden D, Kandoi S, Nagel P, Mavrikakis M, Dumesic JA. Microkinetic analysis and mechanism of the water gas shift reaction over copper catalysts. Journal of Catalysis 2011 7/1;281(1):1-11.

[65] Callaghan CA, Vilekar SA, Fishtik I, Datta R. Topological analysis of catalytic reaction networks: Water gas shift reaction on $\mathrm{Cu}(111)$. Applied Catalysis A: General 2008 $8 / 1 ; 345(2): 213-32$.

[66] Grabow LC, Gokhale AA, Evans ST, Dumesic JA, Mavrikakis M. Mechanism of the Water Gas Shift Reaction on Pt: First Principles, Experiments, and Microkinetic Modeling. J. Phys. Chem. C 2008;112(12):4608-17.

[67] Mhadeshwar AB, Wang H, Vlachos DG. Thermodynamic consistency in microkinetic development of surface reaction mechanisms. J. Phys. Chem. B 2003;107(46):12721-33.

[68] Grabow LC, Mavrikakis M. Mechanism of Methanol Synthesis on Cu through $\mathrm{CO}_{2}$ and CO Hydrogenation. ACS Catalysis 2011;1(4):365-84.

[69] Wang S, Temel B, Shen J, Jones G, Grabow LC, Studt F, et al. Universal BrønstedEvans-Polanyi Relations for $\mathrm{C}-\mathrm{C}, \mathrm{C}-\mathrm{O}, \mathrm{C}-\mathrm{N}, \mathrm{N}-\mathrm{O}, \mathrm{N}-\mathrm{N}$, and $\mathrm{O}-\mathrm{O}$ Dissociation Reactions. Catalysis Letters 2011;141(3):370-3.

[70] Maestri M, Reuter K. Semiempirical Rate Constants for Complex Chemical Kinetics: First-Principles Assessment and Rational Refinement. Angewandte Chemie, International Edition 2011;50(5):1194-7.

[71] Maestri M, Reuter K. Molecular-level understanding of WGS and reverse WGS reactions on $\mathrm{Rh}$ through hierarchical multiscale approach. Chemical Engineering Science 2012;74:296-9.

[72] Mhadeshwar AB, Vlachos DG. A catalytic reaction mechanism for methane partial oxidation at short contact times, reforming, and combustion, and for oxygenate decomposition and oxidation on Platinum. Ind. Eng. Chem. Res. 2007;46(16):5310-24.

[73] Shustorovich E, Sellers H. The UBI-QEP method: A practical theoretical approach to understanding chemistry on transition metal surfaces. Surface Science Reports 1998;31(16):1-119.

[74] Greeley J, Mavrikakis M. Competitive paths for methanol decomposition on Pt(111). JACS 2004;126(12):3910-9. 
[75] Anton AB, Cadogan DC. The mechanism and kinetics of water formation on $\mathrm{Pt}(111)$. Surf.Sci. 1990 12/2;239(3):L548-60.

[76] Kua J, Goddard III WA. Oxidation of methanol on 2nd and 3rd row group VIII transition metals (Pt, Ir, Os, $\mathrm{Pd}, \mathrm{Rh}$, and $\mathrm{Ru}$ ): Application to direct methanol fuel cells. J. Am. Chem. Soc. 1999;121(47):10928-41.

[77] Acharya CK, Turner $\mathrm{CH}$. CO oxidation with $\mathrm{Pt}(111)$ supported on pure and borondoped carbon: A DFT investigation. Surf.Sci. 2008 12/1;602(23):3595-602.

[78] Chen Y, Vlachos DG. Hydrogenation of ethylene and dehydrogenation and hydrogenolysis of ethane on $\operatorname{Pt}(111)$ and $\operatorname{Pt}(211)$ : A Density Functional Theory study. J. Phys. Chem. C 2010;114(11):4973-82.

[79] Michaelides A, Hu P. A Density Functional Theory study of the reaction of C+O, $\mathrm{C}+\mathrm{N}$, and $\mathrm{C}+\mathrm{H}$ on close packed metal surfaces. J. Chem. Phys. 2001;114(13):5792-5.

[80] Kua J, Goddard III WA. Chemisorption of organics on Platinum. 2. Chemisorption of $\mathrm{C}_{2} \mathrm{H}_{\mathrm{x}}$ and $\mathrm{CH}_{\mathrm{x}}$ on $\mathrm{Pt}(111)$. J. Phys. Chem. B 1998;102(47):9492-500.

[81] Windham RG, Bartram ME, Koel BE. Coadsorption of ethylene and potassium on $\mathrm{Pt}(111)$. 1. Formation of a pi-bonded state of ethylene. J. Phys. Chem. 1988;92(10):286270.

[82] McMaster MC, Arumainayagam CR, Madix RJ. Molecular propane adsorption dynamics on Pt(111). Chem. Phys. 1993;177(2):461-72.

[83] Li S, Petzold L. Software and algorithms for sensitivity analysis of large-scale differential algebraic systems. J.Comput.Appl.Math. 2000 12/15;125(1-2):131-45.

[84] Hartmann M, Maier L, Minh HD, Deutschmann O. Catalytic partial oxidation of isooctane over rhodium catalysts: An experimental, modeling, and simulation study. Combust.Flame 2010 9;157(9):1771-82.

[85] Prasad V, Karim Av, Arya A, Vlachos DG. Assessment of Overall Rate Expressions and Multiscale, Microkinetic Model Uniqueness via Experimental Data Injection: Ammonia Decomposition on $\mathrm{Ru} / \gamma-\mathrm{Al}_{2} \mathrm{O}_{3}$ for Hydrogen Production. Industrial and Engineering Chemistry Research 2009;48(11):5255-65.

[86] Prasad V, Vlachos DG. Multiscale Model and Informatics-Based Optimal Design of Experiments: Application to the Catalytic Decomposition of Ammonia on Ruthenium. Industrial and Engineering Chemistry Research 2008;47(17):6555-67.

[87] Mhadeshwar AB, Kitchin JR, Barteau MA, Vlachos DG. The role of adsorbateadsorbate interactions in the rate controlling step and the most abundant reaction intermediate of $\mathrm{NH}_{3}$ decomposition on Ru. Catal. Lett. 2004;96(1-2):13-22. 
[88] Basini L, Sanfilippo D. Molecular aspects in syn-gas production: The $\mathrm{CO}_{2}$-reforming reaction case. Journal of Catalysis 1995 11;157(1):162-78.

[89] Gustafson BL, Walden JV, inventors. AnonymousConversion of carbon dioxide to carbon monoxide. . 1991 .

[90] Morley C. Gaseq: A chemical equilibirum program for Windows, version 0.79, http://www.cmorley.dsl.pipex.com/.

[91] Wei J, Iglesia E. Mechanism and Site Requirements for Activation and Chemical Conversion of Methane onSupported Pt Clusters and Turnover Rate Comparisons among Noble Metals. Journal of Physical Chemistry B 2004;108(13):4094-103.

[92] Salciccioli M, Stamatakis M, Caratzoulas S, Vlachos DG. A review of multiscale modeling of metal-catalyzed reactions: Mechanism development for complexity and emergent behavior. Chemical Engineering Science 2011 10/1;66(19):4319-55. 